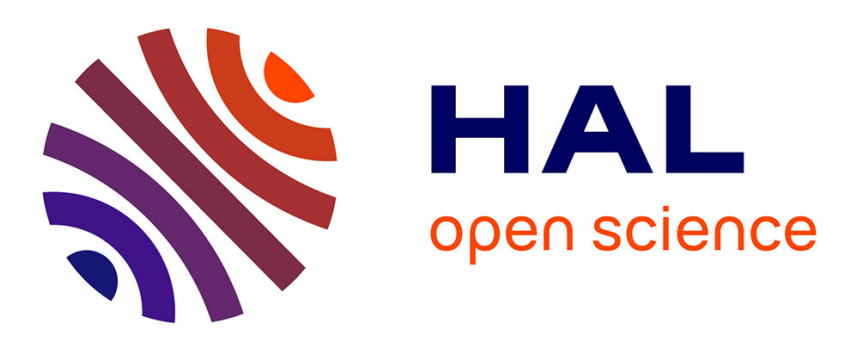

\title{
Nested sequences of hippocampal assemblies during behavior support subsequent sleep replay
}

\author{
Céline Drieu, Ralitsa Todorova, Michaël Zugaro
}

\section{To cite this version:}

Céline Drieu, Ralitsa Todorova, Michaël Zugaro. Nested sequences of hippocampal assemblies during behavior support subsequent sleep replay. Science, 2018, 362 (6415), pp.675-679. 10.1126/science.aat2952 . hal-02330673

\section{HAL Id: hal-02330673 https://hal.science/hal-02330673}

Submitted on 24 Oct 2019

HAL is a multi-disciplinary open access archive for the deposit and dissemination of scientific research documents, whether they are published or not. The documents may come from teaching and research institutions in France or abroad, or from public or private research centers.
L'archive ouverte pluridisciplinaire HAL, est destinée au dépôt et à la diffusion de documents scientifiques de niveau recherche, publiés ou non, émanant des établissements d'enseignement et de recherche français ou étrangers, des laboratoires publics ou privés. 


\title{
Nested Sequences of Hippocampal Assemblies During Behavior Support Subsequent Sleep Replay
}

\author{
Céline Drieu, ${ }^{1}$ Ralitsa Todorova, ${ }^{1}$ Michaël Zugaro, ${ }^{1 *}$ \\ ${ }^{1}$ Center for Interdisciplinary Research in Biology (CIRB), \\ Collège de France, CNRS, INSERM, PSL Research University, Paris, France \\ ${ }^{*}$ Corresponding author. E-mail: michael.zugaro@college-de-france.fr
}

Consolidation of spatial and episodic memories is thought to rely on replay of neuronal activity sequences during sleep. However, the network dynamics underlying the initial storage during wake have never been tested. While slow, behavioral timescale sequences have been claimed to sustain sequential memory formation, fast ('theta') timescale sequences, nested within slow sequences, could be instrumental. We found that during passive travel, place cells formed behavioral timescale sequences but theta sequences were degraded, resulting in impaired subsequent sleep replay. In contrast, when the rats actively ran during transportation, place cells generated clear theta sequences and accurate trajectory replay during sleep. Our results support the view that nested sequences underlie the initial formation of memory traces subsequently consolidated during sleep.

The sequential activation of neuronal ensembles is a ubiquitous brain coding scheme possibly underlying numerous diverse behaviors (1-4). Sequential neuronal activity occurs at different timescales, ranging from slow ('behavioral') timescales, where the dynamics are constrained by stimulus or motor time constants, to fast ('endogenous') timescales mostly driven by intrinsic network properties. A prominent example is the hippocampus, where place cells code for an animal's location (5). As the animal explores its environment, different place cell ensembles become successively active along the ongoing trajectory, yielding sequences of neuronal activity at the behavioral timescale. During subsequent slow wave sleep (SWS), the same sequences are endogenously replayed during sharp-wave ripple (SWR) complexes, at a highly accelerated $(20 \times)$ timescale (6), mediating memory consolidation during sleep $(7-10)$. How is the sequential organization of place cell assemblies maintained across these two timescales expressed at entirely disjoint moments in time and during different brain states?

Sequential information could be readily stored during exploration in the hippocampal network by the sequential activation of place cells at the behavioral timescale, via a recently uncovered form of behavioral timescale plasticity (11). An intriguing alternative is that sequential structure is stored via the remarkable property of the hippocampal network to generate nested sequences of cell assemblies, whereby both slow and fast neural sequences are intermingled in time. This occurs during spatial navigation: nested within behavioral timescale sequences, the hippocampal network also produces sequences at the 'theta' timescale (one sequence per theta cycle of $\sim 150 \mathrm{~ms},(12-14)$ ) allowing cell assemblies to fire within brief delays $(\sim 25 \mathrm{~ms},(15))$ compatible with classical Hebbian plasticity, such as spike-timing dependent plasticity (16). Are these nested sequences of hippocampal cell assemblies required for subsequent sleep replay $(12,13)$, or are they but a stunning epiphenomenon, deriving from preexisting connectivity within the hippocampal network (17-19)?

Contrasting these predictions requires a protocol that selectively disrupts fast, theta sequences, but preserves slow, behavioral sequences of place cell assemblies. Further crucial constraints are the ability to trigger or release theta sequence disruption with temporal precision, as well as the necessity to target the entire hippocampal formation, both in terms of extent (to overcome information redundancy along the septo-temporal axis of the hippocampus), and in terms of fields (to overcome compensatory network mechanisms such as pattern completion in CA3, which could restore locally induced impairments). 
During passive transportation in space, place cells remain spatially selective, but their precise timing relative to theta (phase precession, (12))) is altered (20), except if the animals actively run on an onboard treadmill (21). We thus transported rats on a model train (fig. S1A) and turned the onboard treadmill off (Passive) or on (Active) to respectively perturb nested sequences or leave them intact. The goal was to determine whether intact nested sequences were required for subsequent replay during
SWS. The rats were tested in an entirely novel environment, different from the training room. Hence, hippocampal activity was monitored as the animals learned a novel spatial context, which is known to induce the formation of a novel hippocampal map, increase network coordination, boost replay, and enhance plasticity (22-25). Further, because the rats were tested on a single day, this avoided any confounding network changes that could have resulted from previous experience.
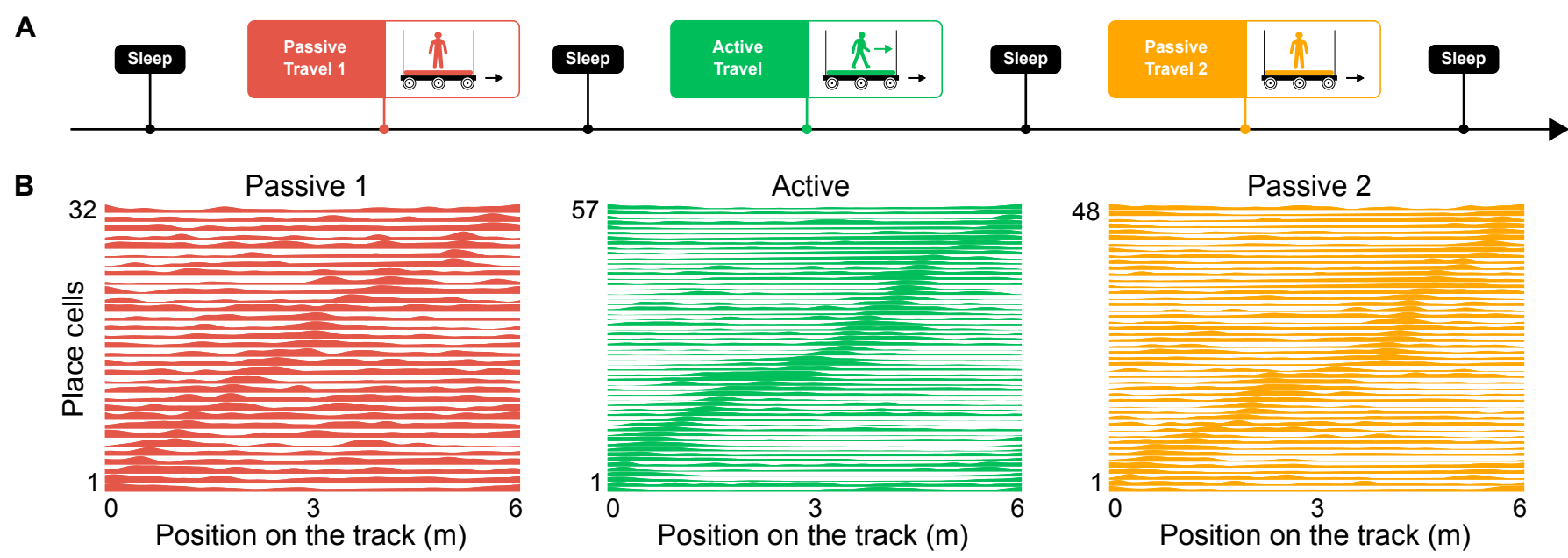

C
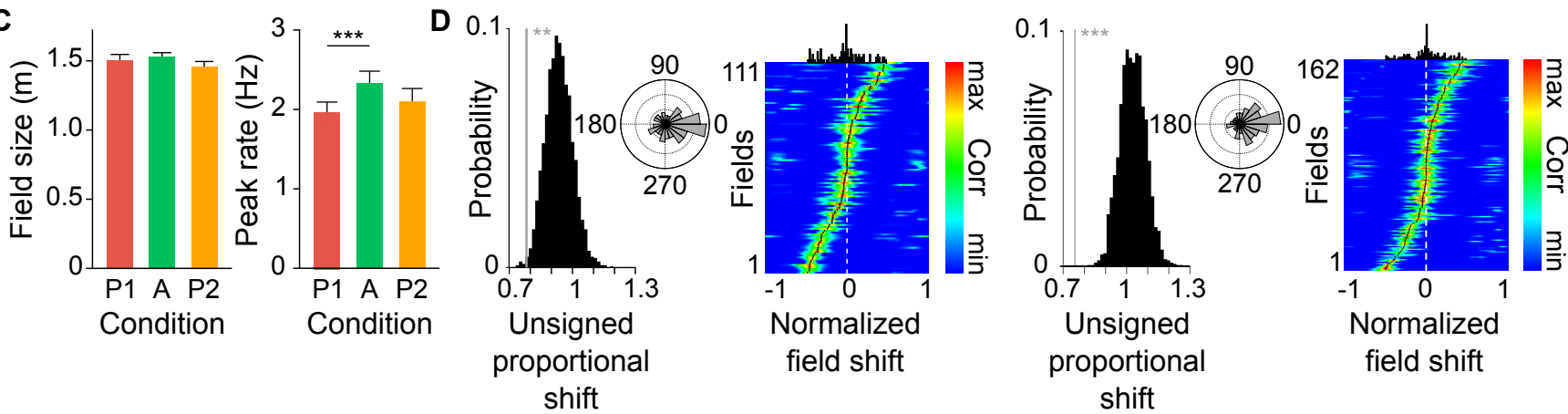

Fig. 1. Maintenance of behavioral timescale properties. (A) Behavioral protocol. In a novel environment, rats underwent three successive travel sessions (Passive 1-Active-Passive 2), interspersed with sleep recordings (see also fig. S1). Passive travel was intended to selectively perturb spike timing at the theta time scale but not at the behavioral timescale. (B) Linearized normalized firing curves of place cells recorded from one example rat across travel sessions, showing complete track coverage in all conditions. (C) Place field size was maintained across travel conditions (Kruskal-Wallis (KW) test, $P>0.05$ ). Peak firing rates were somewhat lower in Passive 1 than in Active (KW test, $* * * P<10^{-4}$ ). (D) Firing fields did not remap between Passive 1 and Active. Left: Mean unsigned proportional shift $\sigma$ (vertical gray lines; ${ }^{* *} P<0.01$ ) and $\sigma$ distribution for $n=2000$ bootstrapped remapping data sets (black histograms). Inset: Circular distributions of angular differences between place field peak locations on the maze (Rayleigh test, $P<0.001$; V-test against $0, P<10^{-4}$ ). Right: Spatial crosscorrelograms of firing fields across successive travel sessions ( $x$ axis normalized to field size; black dots represent correlogram modes; black histograms at top, mode distributions). (E) Firing fields also did not remap between Active and Passive 2. Data are displayed as in (D). Left: ${ }^{* * *} P \sim 0$. Inset: Rayleigh test, $P<10^{-9}$; V-test against $0, * P<10^{-11}$. 
We recorded CA1 pyramidal units and local field potentials in five rats. Following a baseline sleep session, the rats underwent three travel sessions (Passive 1, Active, Passive 2) interspersed with sleep sessions (Fig. 1A). The train velocity, number of laps and recording duration were similar in all conditions for all rats (Fig. S1B-D). We first verified the presence of place cell sequences at the behavioral timescale in all three conditions. Pyramidal cells always coded for the location of the animal in space (Figs. 1B and S2). Their fields remained similar in terms of size (Figs. 1C and S3), and together covered the entire train track (Fig. S2C). However, peak firing rates and place cell count were
A

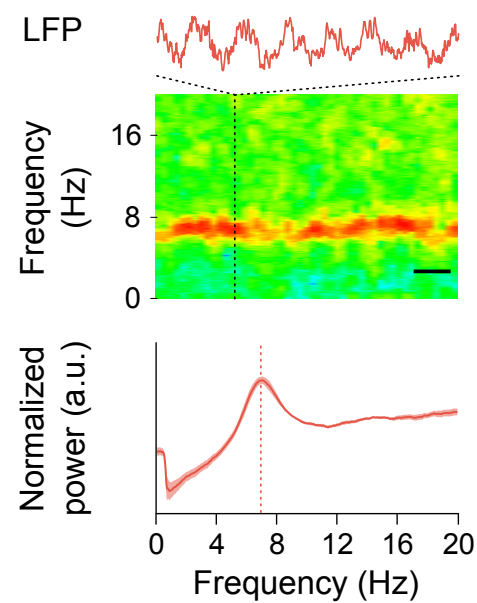

C

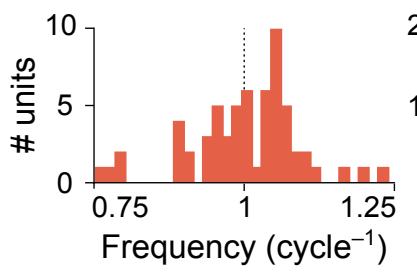

E

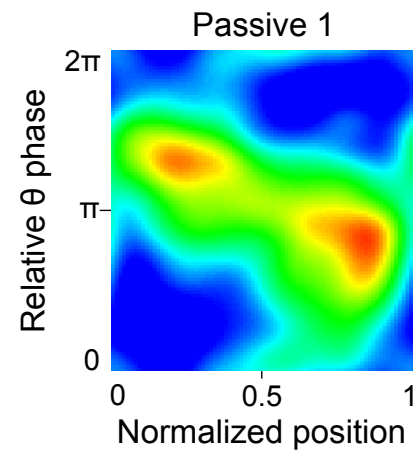

Active
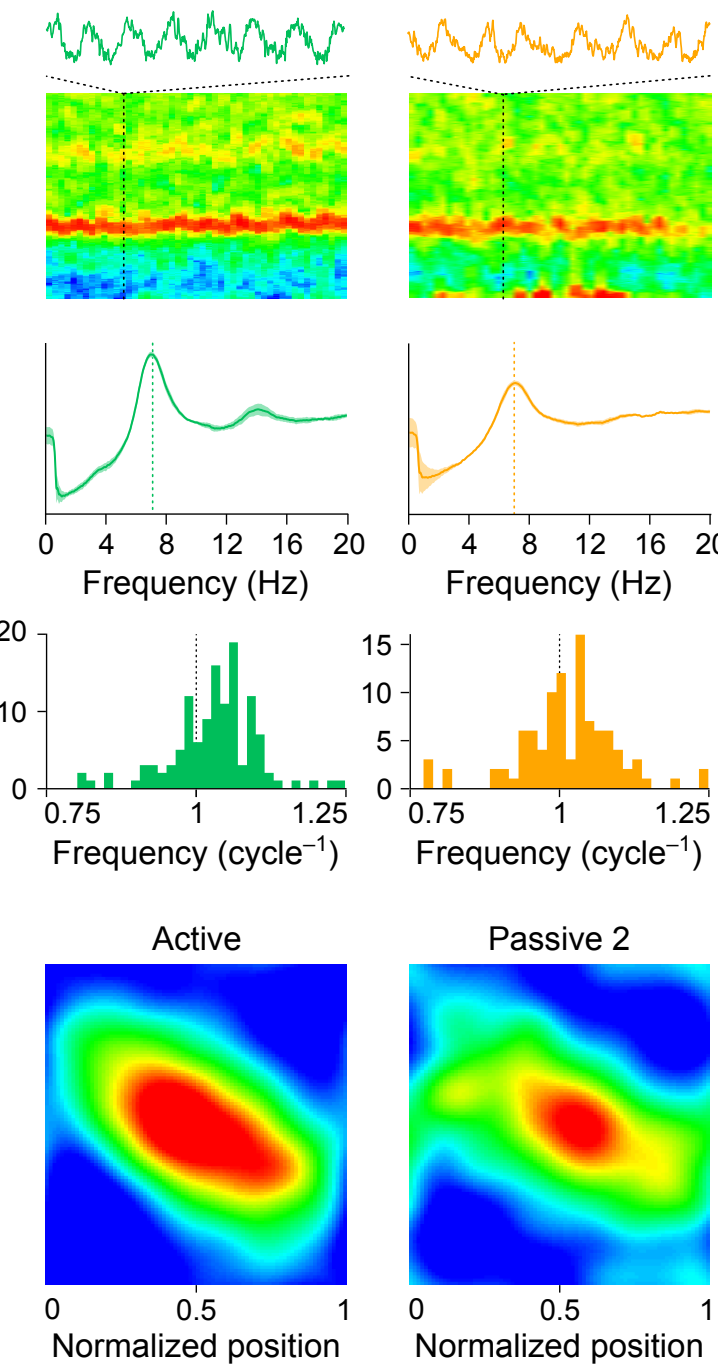

Passive 2
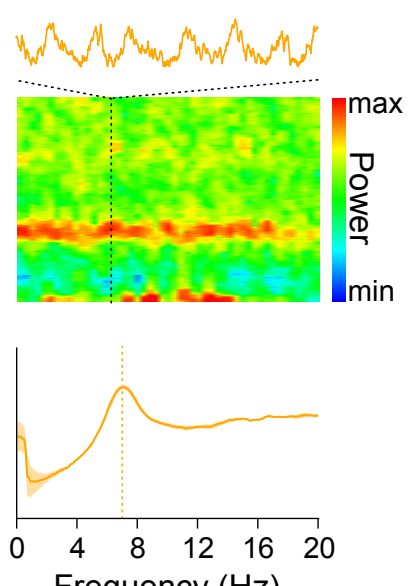

Frequency $(\mathrm{Hz})$
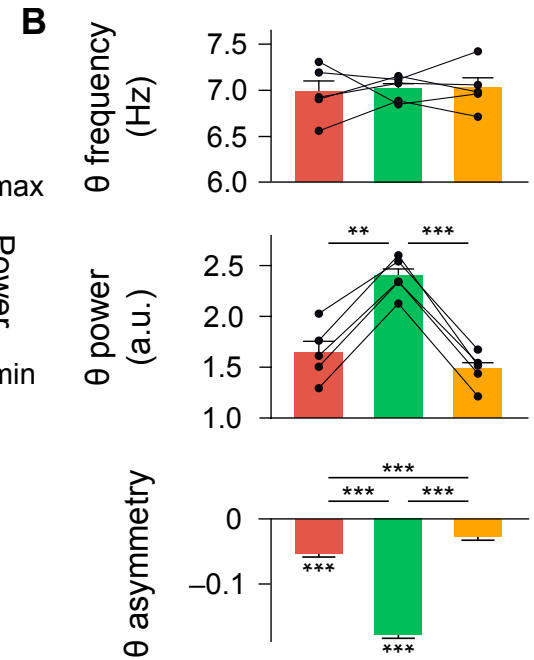

D

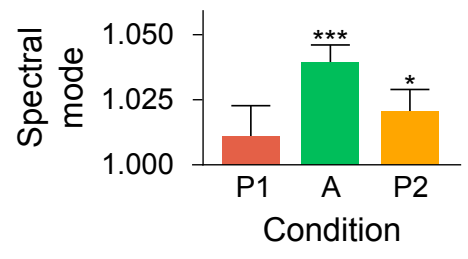

F

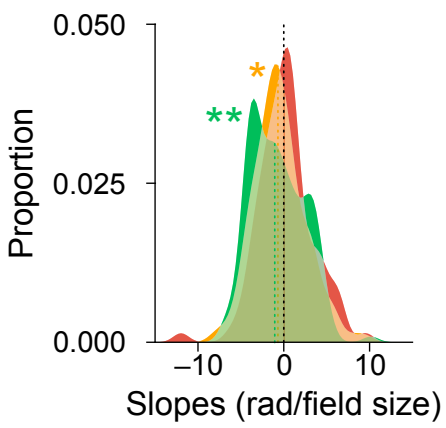

Fig. 2. Perturbation of single-cell theta timescale properties. (A) Theta maintenance across travel sessions. Top row: Example raw LFPs (duration, $1 \mathrm{~s}$ ). Middle row: Power spectrograms (black dashed line, time of LFP traces shown above; black calibration bar, $15 \mathrm{~s}$ ). Bottom row: Normalized power spectra (mean \pm SEM). (B) Theta frequency was unchanged across conditions [top; repeated-measures analysis of variance (ANOVA), $P>0.90$ ], while theta power (middle; repeated-measures ANOVA, $P<10^{-6}$ ) and shape (bottom, theta asymmetry; $\mathrm{KW}$ test, $P<10^{-84}$ ) were altered during passive travel (frequency and power, mean $\pm \mathrm{SEM}$ ). ${ }^{* *} P<0.0033$, ${ }^{* * *} P<0.00033$ for post hoc comparisons; ${ }^{* *} P<0.01,{ }^{* * *} P<0.001$ otherwise. (C-D) During passive travel, spike bursts recurred at lower rates, closer to baseline theta frequency. (C) Distributions of spectral modes of spike trains (measured relative to theta frequency, $x$ axis trimmed at $\pm 25 \%$ around theta frequency). (D) Spectral modes $\left(P<10^{-6}, * P<0.02\right)$. (E-F) Theta phase precession was perturbed during passive travel. (E) Average normalized phase precession density plots for significantly phase-precessing cells (blue and red indicate minimum and maximum spike density, respectively). (F) Distribution of phase precession slopes for all place cells (KS test, ${ }^{* *} P<0.008, * P<0.04$; colored dashed vertical lines are medians). 
somewhat reduced during passive travels (Figs. 1C and S2B; (20)); specific controls for these factors will be provided in ensuing analyses. Incidentally, we also confirmed that place fields did not undergo random remapping (Figs. 1D and S2D). This further supported that hippocampal dynamics were virtually identical at the slow timescale in all travel sessions.

Clear theta oscillations with similar frequencies were observed in all conditions (Fig. 2A). However, during passive travel, power was slightly reduced at both the fundamental frequency and first harmonic, the latter resulting in decreased cycle asymmetry, indicative of a change in the internal structure of theta cycles (Fig. 2A-B). This was expected to alter the precise spike timing of active cell assemblies. Consistently, while place cells continued to oscillate slightly faster than theta ('phase precession') during active travel, this was reduced in passive travel (Figs. 2C-D and S4A-B), indicating an overall degradation of phase precession (Fig. 2E$\mathrm{F}$; in phase precessing neurons, phase range was similar in all conditions (26), Fig. S4C-E). This degradation was noteworthy, because phase precession is thought to be instrumental for the formation of nested sequences: because place cells normally oscillate slightly faster than theta, they emit spike bursts earlier and earlier in successive theta cycles; this (possibly combined with additional coordinating mechanisms $(27,28)$ ) results in newly activated cells to fire after those that have started firing in earlier cycles, effectively resulting in temporal sequences of activity.

To directly assess how perturbation of phase precession affected theta sequences, we used a Bayesian reconstruction approach $(21,29,30)$. Briefly, theta cycles were subdivided in 6 phase bins, and the sequential structure of reconstructed positions in these bins ('candidate events') was evaluated using two previously described complementary measures (30): trajectory scores (normalized to compare across animals and conditions, see Methods), which assess the quality (linearity) of the reconstructed events, i.e. whether the events represent spatially aligned positions vs mere series of random locations; and slopes, which estimate the speed at which reconstructed events are played and indicate whether the events do move through space vs remain merely stationary (absence of actual trajectories). Thus, clear theta sequences would be characterized by both high scores and slopes, whereas static representations of current position would result in high scores but low or zero slopes, and random activity would be associated with low scores (but possibly spuriously high slopes). Clear sequential structure was readily visible in individual theta cycles during active, but not passive, travel (Fig. 3A). This was confirmed over all theta cycles for all rats (Figs. 3B and S5). While normalized trajectory scores were significantly better than chance in all conditions (Fig. 3C), slopes were significantly steeper during active travel (Figs. 3D and S5C-D). Joint analysis of trajectory scores and slopes revealed a much greater proportion of high-value pairs during active travel (Fig. 3E). To confirm and extend these results using an independent measure, we computed the quadrant score (27) of each candidate event, which assessed the overall direction of reconstructed trajectories without assuming constant velocity. Quadrant scores were significantly greater for active travel (clear trajectories), and remained very low for passive travel (degraded trajectories) (Figs. 3F and 5B).

Hence, theta sequences were degraded during passive travel. Note that reconstruction quality and quadrant scores were higher in Passive 2 than in Passive 1 (Fig. 3E-F). This argues that sequential structure was slightly less degraded in the second passive travel session (Fig. 3B). This is further supported by higher self-consistency of theta sequences (see Methods) in Passive 2 compared to Passive 1, while Active sequences were the most self-consistent (Figs. 3G and S6A).

We controlled that our results could not be accounted for by differences in the number of simultaneously recorded place cells (Fig. S7) and their firing rates (Fig. S8). We also controlled for decoding quality (see Methods and Fig. S9), ruling out a potential bias due to differences in spatial coding, both at the single cell and at the population level. Finally, because small variations in field locations between individual laps could have altered sequence detection, we also ruled out differences in firing variability between travel conditions (see Methods; Fig. S10).

Taken together, the above results show that place cell sequences at the behavioral timescale were present in all three conditions, but theta sequences were disrupted during passive travel, when stationary network activity continued to reflect the ongoing position at the endogenous timescale. How did this impact subsequent activity during slow wave sleep? 
A
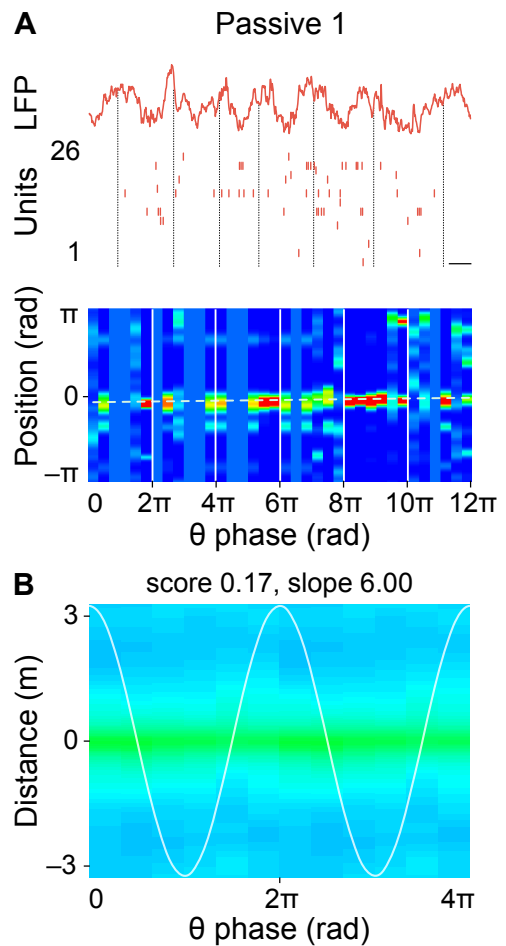

C

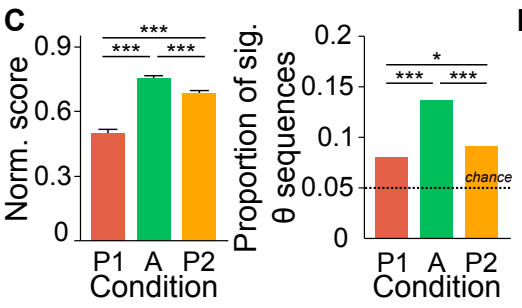

E

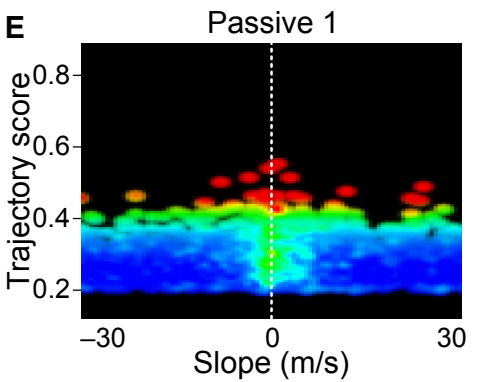

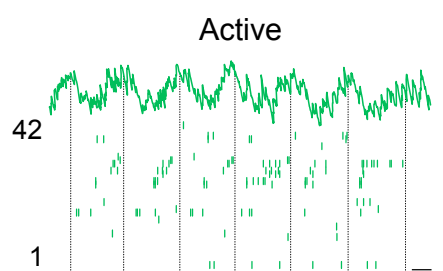
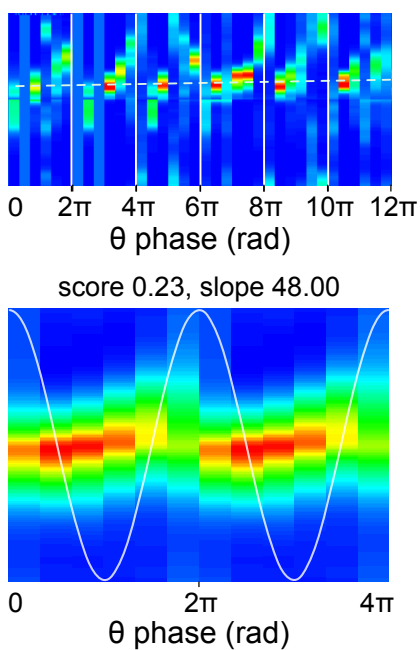

D
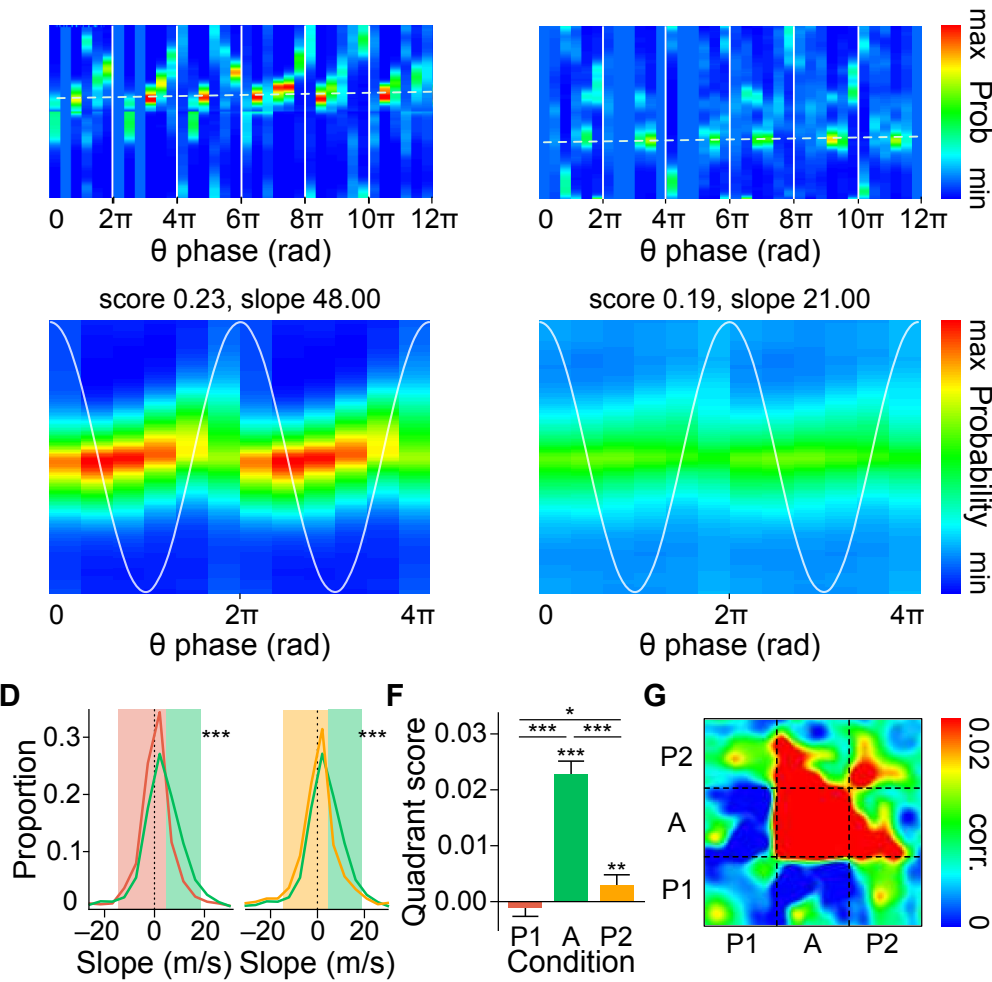

score 0.19 , slope 21.00

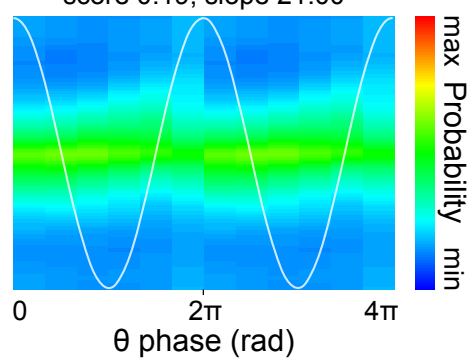

G
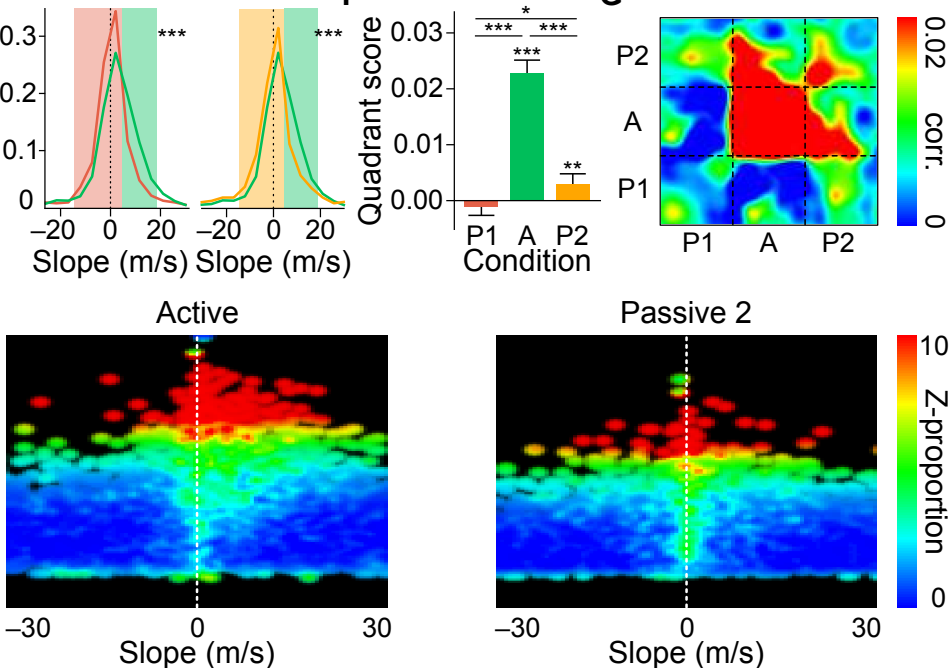

Fig. 3. Perturbation of theta sequences. (A) Top: Raw LFPs and place cell spikes in 6 example theta cycles (black dashed lines, theta peaks; place cells are ordered by their place field location on the track) in each travel condition (black calibration bars, $50 \mathrm{~ms}$ ). Bottom: Bayesian reconstruction of position encoded in the ongoing activity of the hippocampal network ( 6 phase windows per theta cycle; white vertical lines, theta peaks; white dashed lines, actual position of the animal). (B) Average Bayesian reconstruction of position (relative to actual position of the animal) across theta subcycles for all rats. Trajectory score and slope (in $\mathrm{cm}_{\text {.cycle }}{ }^{-1}$ ) are indicated above each reconstruction. Two cycles are shown for clarity. (C) Left: Normalized score of theta sequences (KW test, ${ }^{* * *} P<10^{-23}$ ). Right: Proportion of significant theta sequences (Passive 1: $7.95 \%$, Active: $13.61 \%$, Passive 2: $9.05 \%$; all proportions are significantly greater than shuffled control proportions, Monte-Carlo test, ${ }^{* * *} P \sim 0$ ). (D) Distributions of significant theta sequence slopes (KS tests; left: Passive 1Active, ${ }^{* * *} P<10^{-16}$; right: Active-Passive $2,{ }^{* * *} P<10^{-18}$ ). Colored bands indicate significant differences. (E) Distribution of theta sequence quality assessed by joint trajectory score and slope (for all animals; color codes proportion normalized relative to shuffled control data). (F) Quadrant score computed from individual theta cycles (KW test, ${ }^{* * *} P<10^{-24} ;{ }^{*} P<0.017$, ***P $<0.00033$ for post hoc comparisons; $* * P<0.01$, $* * * P<0.001$ otherwise). (G) Pairwise bias correlation between awake theta sequences (ordered according to time of occurrence). Sequences were stable only during active travel. Note absence of correlation during Passive 1, and progressive decay following Active during Passive 2. 
Candidate replay events were defined as transient surges in aggregate firing rate (30) during SWS epochs (Fig. S11C), which coincided with ripple events (Fig. S11F-G; results remained unchanged when candidate events were restricted to ripples, Fig. S12). The average SWS duration, number of candidate events and ripple occurrence rate were not significantly different across animals (Figs. S11A-F). To reconstruct replayed trajectories, the Bayesian decoder was first trained using place cell activity recorded during the preceding travel session, then tested during SWS on candidate events subdivided into $20 \mathrm{~ms}$ non-overlapping time windows (see Methods).
A

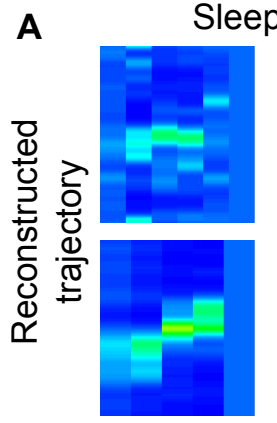

Time (20 ms bins)
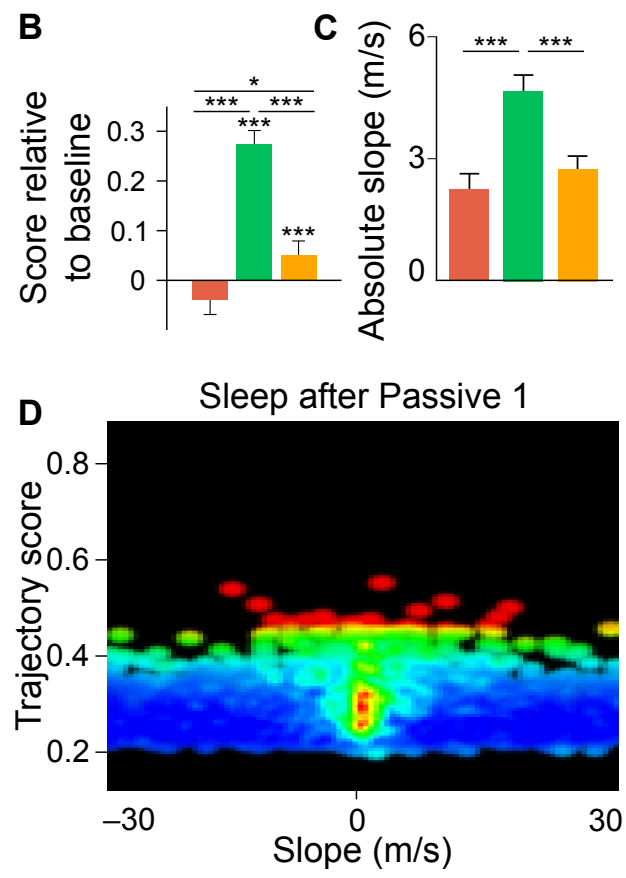

Sleep after Active

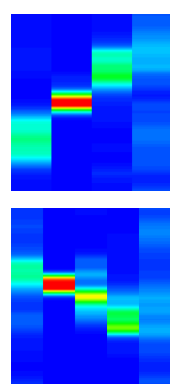

Time (20 ms bins)
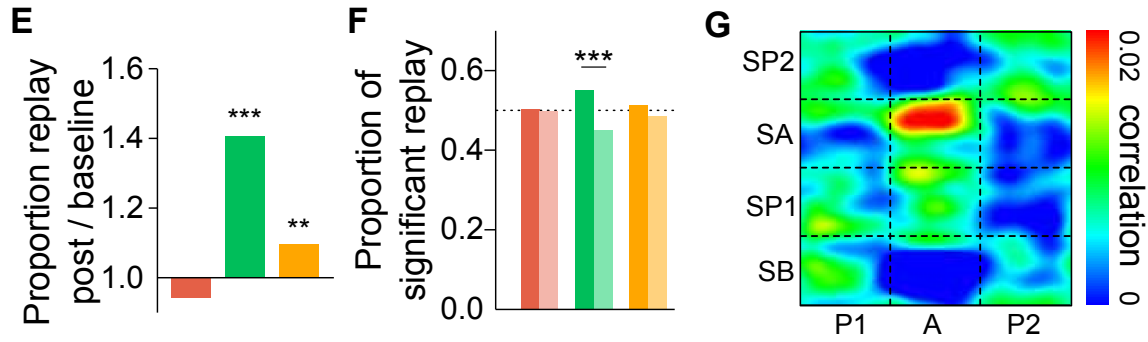

Sleep after Passive 2
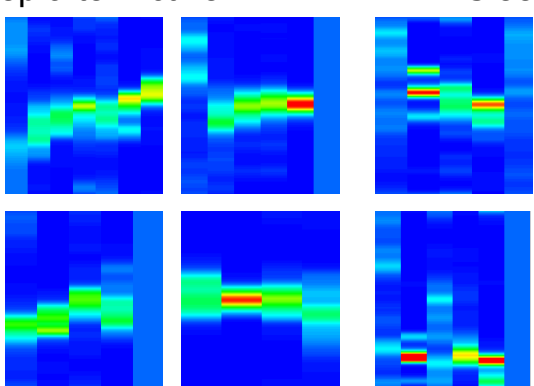

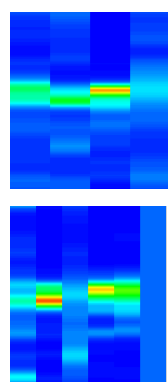

Time (20 ms bins)
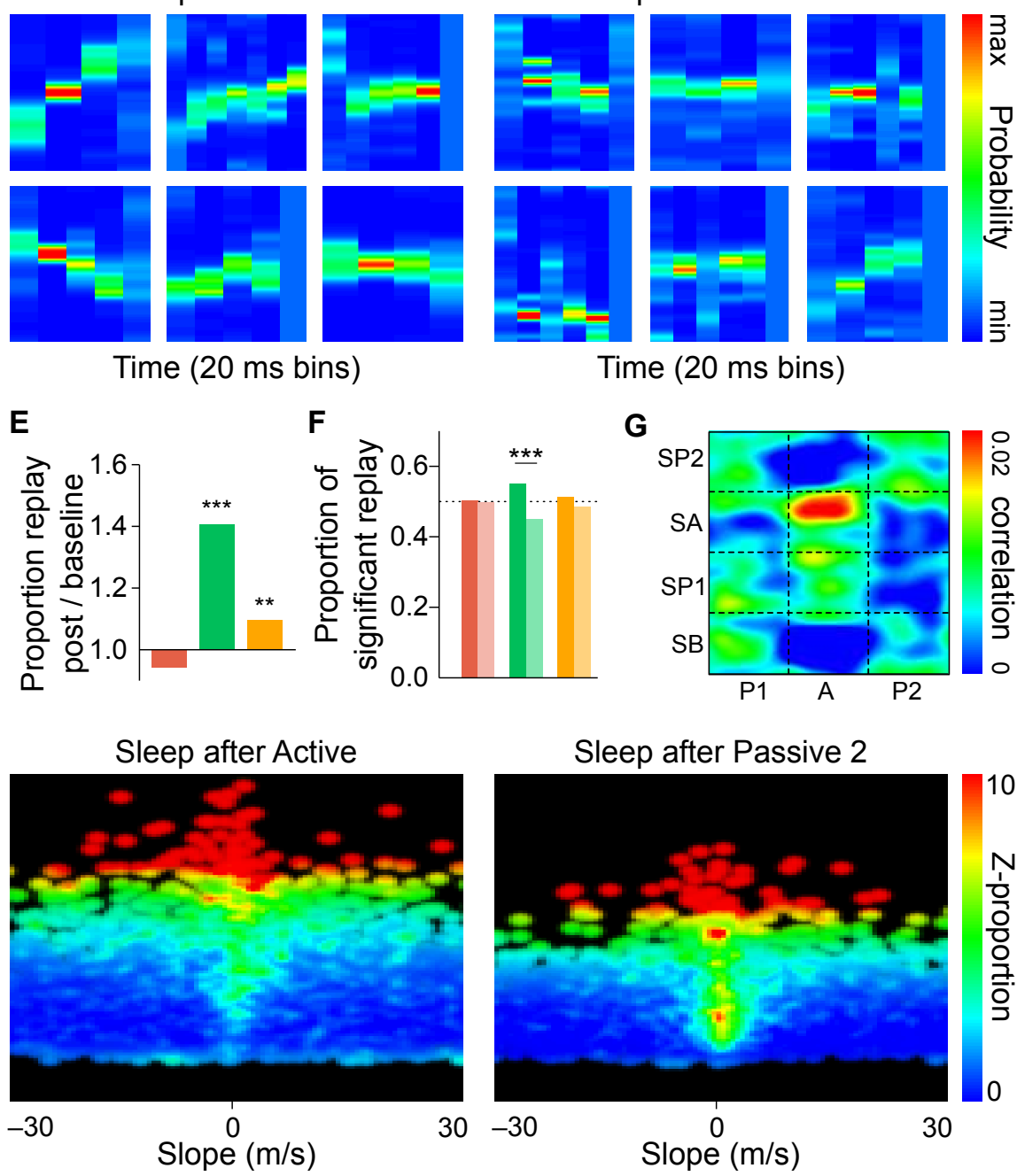

Fig. 4. Replay is degraded following perturbation of theta sequences. (A) Examples of significant replay events in sleep sessions (see fig. S13 for more examples). (B) Scores of replay events relative to baseline sleep (KW test, ${ }^{* * *} P<10^{-17} ;{ }^{*} P<0.017,{ }^{* * *} P<0.00033$ for post hoc comparisons; ${ }^{* *} P<0.01,{ }^{* * *} P<0.001$ otherwise). (C) Absolute slopes of replay events (KW test, ${ }^{* * *} P<10^{-27}$ ). (D) Distribution of replay quality assessed by joint trajectory score and slope (for all animals; color codes for proportion normalized relative to shuffled control data). (E) Proportion of significant replay events relative to baseline sleep (binomial proportion tests, ${ }^{* *} P<0.01,{ }^{* * *} P<0.001$ ). Sleep replay was boosted following active travel, compared to after passive travel. (F) Proportion of forward (darker colors) vs reverse (lighter colors) replay events (binomial proportion tests, ${ }^{* * *} P<0.001$ ). (G) Pairwise bias correlation between awake theta sequences and sleep replay (ordered according to time of occurrence).

Candidate replay events were evaluated using the same method as theta sequences, whereby genuine sequences are characterized by both high tra- jectory scores and slopes. While candidate events with significant trajectory scores were present in all three sleep sessions, overall the reconstructed 
trajectories were notably sharper following active travel (Figs. 4A and S13A), and scores were significantly improved relative to baseline (see Methods) only in sleep sessions following Active and Passive 2 (Fig 4B). In addition, slopes were significantly steeper following Active (Figs. 4C and S13B). Joint analysis of trajectory scores and slopes confirmed a much greater proportion of high value pairs following active travel (Figs. 4D and S13C-D).

We next addressed the critical question of whether these trajectories did constitute genuine replay of awake behavior, or merely reflected preexisting connectivity patterns independent of experience. We compared the proportion of significant trajectories in each sleep session relative to baseline sleep, i.e. relative to the sleep session preceding any exploration of the environment (as pointed out above, recordings took place in an entirely novel environment). We did not observe replay during sleep following Passive 1 (Fig. 4E), when theta sequences had been selectively disrupted (Fig. 3). Trajectory replay was then boosted in sleep following Active (Fig. 4E), when nested sequences had remained intact (Fig. 3). Finally, an intermediate but significant level of reactivation was observed following Passive 2 (Fig. 4E), when theta sequences had been perturbed to a lesser degree than during Passive 1 (Fig. 3). Interestingly, while forward and backward trajectories were found in equal proportions during sleep following passive travel, only active travel resulted in a greater proportion of forward replay actually reflecting awake experience (Fig. 4F). Finally, a direct comparison of theta and replay sequences highlighted a selective correlation between theta sequences in Active and candidate replay events in subsequent sleep (Figs. 4G and S6B-C).

Our results thus show that during sleep following selective disruption of theta sequences (Passive 1), the proportion of significant trajectories remained at baseline levels observed prior to experience. By contrast, intact nested sequences (Active) resulted in boosted replay during sleep, and trajectories in hippocampal space were preferentially replayed in the same direction as in physical space. In Passive 2, theta sequences were perturbed to a lesser degree than in Passive 1, yielding intermediate levels of trajectory replay during subsequent sleep. This has three implications. First, repeated experience alone cannot account for the improved replay following Active, because replay was degraded following Passive 2. Second, the improved theta sequences during Passive 2 compared to Passive 1 are consistent with the notion that replay following Ac- tive resulted in consolidation (7-10), possibly consisting of functional network changes $(12,13)$, that carried over to subsequent sessions. Third, during Passive 2, scrambled activation of place cells at the theta timescale appears to have interfered with previously formed and consolidated memory traces (during Active and subsequent sleep), resulting in degraded replay during sleep after Passive 2.

How would nested sequences be altered during passive travel? In the absence of active locomotor signals, spike bursts of pyramidal cells recurred at slightly longer time intervals, consistent with the fact that bursting frequency increases with running speed $(12,31)$. As predicted by theoretical models $(13,14,32,33)$, this decrease in oscillation frequency resulted in impaired theta phase precession and prevented the formation of theta sequences. Theta sequences have been related to memory performance $(34,35)$, although the underlying mechanisms have remained unclear. Our results indicate a causal link between theta sequences and sleep replay for memory consolidation, and suggest that behavioral timescale sequences are insufficient to store sequential information for reactivation during subsequent sleep. Nested sequences, emerging from independently phase precessing place cells $(13,33)$, enabled hippocampal assemblies to fire dozens of milliseconds apart, which is optimal for classical plasticity mechanisms (16) and can reinforce synaptic connections $(13,14)$. This would effectively store sequential organization as network connectivity patterns, which can later be replayed during sleep for long term consolidation $(7-10)$. Spatio-temporal spike patterns supporting nested sequences have also been reported in the striatum (36) and medial prefrontal cortex (37). This may represent a general neural mechanism to encode and store initial memory traces, and plan future actions $(34,38)$.

Acknowledgments We thank D. Robbe for comments on an earlier version of the manuscript, Y. Dupraz for technical support, and A.C. Segú for help in animal training. This work was supported by the Agence Nationale de la Recherche (ANR-15-CE16-0001-02), French Ministry of Research (C.D.), Collège de France (C.D.), and a joint grant from École des Neurosciences de Paris Île-de-France and LabEx MemoLife (ANR-10-LABX-54 MEMO LIFE, ANR10-IDEX-0001-02 PSL*) (R.T.). The authors declare no competing interests. C.D. and M.Z. designed the study. C.D. performed the experiments. C.D., R.T. and M.Z. designed the analyses. C.D. and R.T. performed the analyses. C.D. and M.Z. wrote the manuscript. Data of this study were stored and curated in servers of the Collège de France, Paris, France. Data of this study are available at: http://dx.doi.org/10.6080/K0Z899MM. This is the author's version of the work. It is posted here by permission of the AAAS for personal use, not for redistribution. The definitive version was published here. 


\section{References}

1. A. J. Peters, S. X. Chen, T. Komiyama, Nature 510, 263 (2014).

2. T. S. Okubo, E. L. Mackevicius, H. L. Payne, G. F. Lynch, M. S. Fee, Nature 528, 352 (2015).

3. K. MacLeod, G. Laurent, Science 274, 976 (1996).

4. E. Pastalkova, V. Itskov, A. Amarasingham, G. Buzsáki, Science (New York, N.Y.) 321, 1322 (2008).

5. J. O'Keefe, L. Nadel, The hippocampus as a cognitive map (Clarendon Press, Oxford, 1978).

6. A. K. Lee, M. A. Wilson, Neuron 36, 1183 (2002).

7. G. Buzsáki, Neuroscience 31, 551 (1989).

8. G. Girardeau, K. Benchenane, S. I. Wiener, G. Buzsáki, M. B. Zugaro, Nature Neuroscience 12, 1222 (2009).

9. V. Ego-Stengel, M. A. Wilson, Hippocampus 20, 1 (2010).

10. N. Maingret, G. Girardeau, R. Todorova, M. Goutierre, M. Zugaro, Nat. Neurosci. 19, 959 (2016).

11. K. C. Bittner, A. D. Milstein, C. Grienberger, S. Romani, J. C. Magee, Science 357, 1033 (2017).

12. J. O'Keefe, M. L. Recce, Hippocampus 3, 317 (1993).

13. W. E. Skaggs, B. L. McNaughton, M. A. Wilson, C. A. Barnes, Hippocampus 6, 149 (1996).

14. G. Dragoi, G. Buzsáki, Neuron 50, 145 (2006).

15. K. D. Harris, J. Csicsvari, H. Hirase, G. Dragoi, G. Buzsáki, Nature 424, 552 (2003).

16. J. C. Magee, D. Johnston, Science (New York, N.Y.) 275, 209 (1997).

17. M. V. Tsodyks, W. E. Skaggs, T. J. Sejnowski, B. L. McNaughton, Hippocampus 6, 271 (1996).

18. G. Dragoi, S. Tonegawa, Nature 469, 397 (2011).

19. A. D. Grosmark, G. Buzsáki, Science 351, 1440 (2016).

20. A. Terrazas, et al., J Neurosci 25, 8085 (2005).

21. A. Cei, G. Girardeau, C. Drieu, K. E. Kanbi, M. Zugaro, Nature Neuroscience 17, 719 (2014).

22. S. Leutgeb, J. K. Leutgeb, A. Treves, M. B. Moser, E. I. Moser, Science 305, 1295 (2004).

23. S. Cheng, L. M. Frank, Neuron 57, 303 (2008).

24. J. O’Neill, T. J. Senior, K. Allen, J. R. Huxter, J. Csicsvari, Nature Neuroscience 11, 209 (2008).

25. S. Li, W. K. Cullen, R. Anwyl, M. J. Rowan, Nature Neuroscience 6, 526 (2003).

26. A. P. Maurer, S. L. Cowen, S. N. Burke, C. A. Barnes, B. L. McNaughton, Hippocampus 16, 785 (2006).

27. T. Feng, D. Silva, D. J. Foster, J. Neurosci. 35, 4890 (2015).

28. S. J. Middleton, T. J. McHugh, Nat. Neurosci. 19, 945 (2016).

29. K. Zhang, I. Ginzburg, B. L. McNaughton, T. J. Sejnowski, J Neurophysiol 79, 1017 (1998).

30. T. J. Davidson, F. Kloosterman, M. A. Wilson, Neuron 63, 497 (2009).

31. C. Geisler, et al., Proceedings of the National Academy of Sciences of the United States of America 107, 7957 (2010).

32. N. Burgess, M. Recce, J. O'Keefe, Neural Networks 7, 1065 (1994).

33. A. Chadwick, M. C. W. van Rossum, M. F. Nolan, ELife 4 (2015).

34. D. Robbe, G. Buzsáki, The Journal of Neuroscience : the Official Journal of the Society for Neuroscience 29, 12597 (2009).

35. Y. Wang, S. Romani, B. Lustig, A. Leonardo, E. Pastalkova, Nat. Neurosci. 18, 282 (2015).

36. M. A. A. van der Meer, A. D. Redish, J. Neurosci. 31, 2843 (2011).

37. M. W. Jones, M. A. Wilson, PLoS Biol 3, e402 (2005).

38. A. M. Wikenheiser, A. D. Redish, Nat. Neurosci. 18, 289 (2015).

39. L. Hazan, M. Zugaro, G. Buzsáki, J Neurosci Methods pp. 207-216 (2006).

40. N. Schmitzer-Torbert, J. Jackson, D. Henze, K. Harris, A. D. Redish, Neuroscience 131, 1 (2005).

41. B. E. Pfeiffer, D. J. Foster, Nature 497, 74 (2013).

42. M. A. Belluscio, K. Mizuseki, R. Schmidt, R. Kempter, G. Buzsáki, The Journal of Neuroscience : the Official Journal of the Society for Neuroscience 32, 423 (2012).

43. R. Kempter, C. Leibold, G. Buzsáki, K. Diba, R. Schmidt, Journal of Neuroscience Methods 207, 113 (2012).

44. Z. J. Roth, Ph.D. thesis, University of Nebraska (2015). 


\section{Materials and Methods}

\section{Animal training}

Five adult male Long-Evans rats (René Janvier, Le Genest Saint Isle, France) weighing 350-400 g were used in this study. Two days after arrival, they were housed individually and maintained on a 12-hour light/dark cycle. Rats were kept on a mild water restriction diet $(>85 \%$ of normal body weight) throughout the training and experiment phases. Critically, training took place in a dedicated room, separate from that where experiments were subsequently carried out. Following one week of daily handling, the rats were first trained to run on a regular treadmill. The speed of the treadmill and the running duration were increased daily over $3-5$ days. In parallel, in order to familiarize the rats with the model train (LGB, Germany), they were placed in the immobile car for 10-15 min sessions. Subsequently, the rats learned to run on the miniature treadmill located inside the (immobile) car, as well as to be passively transported by the train (treadmill off), before they finally learned to run on the miniature treadmill while being transported by the train ( $\sim 3$ weeks; Fig. S1A). The rats were always transported in the forward direction at constant speed $\left(\sim 45-50 \mathrm{~cm} . \mathrm{s}^{-1}\right.$, fig. S1B $)$ on the $2.20 \mathrm{~m} \times 1.30 \mathrm{~m}$ obround-shaped track. The treadmill and the train velocity were controlled by the same generator. The rats received sucrose water rewards ( $1.25 \%$ saccharine), delivered by a solenoid valve in a fountain placed at the front of the car (fig. S1A, right) whenever the car arrived at one of two different reward locations on the track. The entire experimental setup was remotely controlled in order to avoid interference with experimenters. All procedures were in accordance with national (Comite d'Ethique \#2011-0008) and international (US National Institutes of Health guidelines) standards and legal regulations (Certificat d'Autorisation d'Exprimenter \#A75-1756) regarding the use and care of animals. Training and experiments took place during the day.

\section{Surgical implantation and electrode ad- justment}

The rats had free access to water for at least two days before surgery. They were deeply anesthetized (xylazine, $0.12 \mathrm{ml}$ intramuscular; pentobarbital, $40 \mathrm{mg}$ per $\mathrm{kg}$ of body weight, intraperitoneal; $0.1 \mathrm{ml}$ pentobarbital supplemented every hour). Sixteen independently movable tetrodes or octrodes (groups of four or eight twisted $13 \mu \mathrm{m}$ tungsten wires, gold-plated to $\sim 150 \mathrm{k} \Omega$ ) were bilaterally implanted above the dorsal hippocampus (3.5 $\mathrm{mm}$ or $4 \mathrm{~mm} \mathrm{AP,} \pm 2.5 \mathrm{~mm}$ ML relative to bregma). Two screws attached to the skull above the cerebellum served as ground and reference, respectively. After a one-week recovery period, the rats were placed back on the mild water restriction diet and retrained in the dedicated training room. The electrodes were progressively lowered into the CA1 pyramidal layer, identified based on both neural firing patterns (e.g. complex spikes) and local field potential (LFP) characteristics (especially sharp wave-ripple complexes).

\section{Behavioural task}

The experiments took place in a separate, novel room. Following a baseline sleep recording session, the rats underwent three transportation sessions: passive (treadmill off), then active (treadmill on), then passive again (fig. 1A). Each travel condition consisted of $2 \times \sim 15$ laps (fig. S1C). During the interval period, the rats stayed for less than $2 \mathrm{~min}$ in a flower pot while the experimenter unrolled the recording tethers. Each travel session was followed by a sleep epoch in the flower pot. Each sleep epoch, including baseline sleep, lasted for $\sim 90$ minutes.

\section{Data acquisition and preprocessing}

For the three rats implanted with sixteen tetrodes, brain signals were preamplified using two 32-channel unity-gain preamplifiers (Noted Bt, Pecs, Hungary) and acquired at 32,552.083 Hz (Digital Lynx, Neuralynx, Bozeman, MO). The head-stages were connected to the recording setup via two custom-made tethers (New England Wire, Lisbon, NH). For the three rats implanted with sixteen octrodes, brain activity was recorded using a 128-channel digital data acquisition system (KJE-1001, Amplipex, Szeged, Hungary). The signals were digitized using two 64channel preamplifiers (Amplipex HS2) and sampled at $20,000 \mathrm{~Hz}$. In both setups, one LED (red) or two LEDs (red and green) were used to track the head of the animal using an overhead video camera (sampling rate $30 \mathrm{~Hz}$, resampled at $39.0625 \mathrm{~Hz}$ ).

Data were pre-processed and visualized using NeuroSuite (http://neurosuite.sourceforge.net, (39)). Briefly, to extract spiking activity, wide-band signals were high-pass filtered (nonlinear median-based filter) and thresholded using NDManager (L. Hazan and M. Zugaro, http://neurosuite.sourceforge.net). Extracted spike waveforms were sorted via a semiautomatic cluster cutting procedure using Klus- 
taKwik (K.D. Harris, http://klustakwik.sourceforge. net) and Klusters (L. Hazan, http://neurosuite. sourceforge.net). Neurophysiological and behavioral data were explored using NeuroScope (L. Hazan, http://neurosuite.sourceforge.net). Units were classified as putative interneurons or pyramidal cells using k-means clustering based on waveform width, waveform trough-to-peak duration, and firing rate. Cluster isolation quality was assessed using the $L_{\text {ratio }}(40,41)$. Briefly, the $L_{\text {ratio }}$ of cluster $C$ is:

$$
L_{\text {ratio }}=\frac{1}{n_{s}} \sum_{i \notin C}\left\{1-C D F_{\chi_{d f}^{2}}\left(D_{i, C}^{2}\right)\right\}
$$

where $n_{s}$ is the total number of spikes recorded on the tetrode throughout the recording epoch, $i \notin C$ is the set of spikes that are not members of cluster $C, D_{i, C}^{2}$ is the Mahalanobis distance of spike $i$ from cluster $C$, and $C D F_{\chi_{d f}^{2}}$ is the cumulative distribution function of the $\chi^{2}$ distribution with $d f=$ number of channels of the electrode $\times$ number of features. Only well-isolated clusters with an $L_{\text {ratio }}<0.05$ were included in the analyses. LFPs were derived from wideband signals by downsampling all channels to $1,250 \mathrm{~Hz}$.

\section{Recording site verification}

Upon completion of the experiments, recording sites were marked with small electrolytic lesions. Two days later, rats were deeply anesthetized with a lethal dose of pentobarbital, and intracardially perfused with saline $(0.9 \%)$ followed by paraformaldehyde (10\%). The desiccated brains were post-fixed and conserved in PFA $(4 \%)$ at $4^{\circ} \mathrm{C}$ until the histology (50 $\mu \mathrm{m}$ coronal slices, Cresyl violet staining).

\section{Data analysis and statistics}

All analyses were performed in Matlab (MathWorks, Natick, MA), using the Freely Moving Animal toolbox (FMAToolbox, M. Zugaro, http:// fmatoolbox.sourceforge.net) and additional custom programs.

Descriptive statistics are reported as mean \pm standard error of the mean when the underlying distribution is Gaussian-shaped (Jarque-Bera test) or median \pm standard error of the median otherwise. Unless indicated otherwise, bars represent median \pm standard error of the median. Repeated measures ANOVA was used for multiple comparisons of paired Gaussian distributions, and differences between groups were assessed using paired Student's t-test with Bonferroni correction for post-hoc analysis. For non-Gaussian distributions of independent (non-paired) data, multiple comparisons were made using Kruskal-Wallis (KW) and differences between groups were assessed using Wilcoxon rank sum test with Bonferroni-correction for post-hoc analysis. For paired data following a non-Gaussian distribution, Friedman test was employed, with signed rank tests with Bonferronicorrection for post-hoc analyses for assessing differences between groups.

Proportions were compared using the binomial proportion test. Medians (of non-Gaussian distributions) were compared to single values with Wilcoxon signed rank tests. Distributions were compared using the Kolmogorov-Smirnov test. No statistical methods were used to pre-determine sample sizes, but our sample sizes are similar to those generally employed in the field. Data collection and analysis were not performed blind to the conditions of the experiments. All statistical tests were two-tailed.

\section{Firing fields}

All analyses of spike trains emitted during the awake state were restricted to epochs in which the linear velocity of the rat was greater than $5 \mathrm{~cm} . \mathrm{s}^{-1}$. Firing maps and linearized firing curves were computed using a kernel-based method (bin size: $2.4 \mathrm{~cm}$ ). The firing rate was estimated at each point $x$ as:

$$
f(x)=\sum n_{t} \times w\left(\left|x-x_{t}\right|\right) / \sum d t \times w\left(\left|x-x_{t}\right|\right)
$$

where, in a given time bin $t, n_{t}$ is the number of action potentials emitted, $x_{t}$ is the position of the rat, and $d t$ is the time bin size. The kernel $w$ was a Gaussian of width $4.5 \mathrm{~cm}$. Firing fields were defined as the set of contiguous bins containing the location of maximal firing rate (min $1 \mathrm{~Hz}$ for Active and $0.7 \mathrm{~Hz}$ for Passive), for which the firing rates exceeded $30 \%$ of the peak firing rate. Pyramidal cells with at least one firing field were defined as place cells.

The stability of firing fields between conditions was assessed using two complementary methods (fig. 1D and fig. S2D). We first tested for random remapping using the method described in (21). Briefly, we measured the unsigned proportional field shifts of all the recorded place cells and compared their average to a null distribution constructed by shuffling $(n=2,000)$ the identity of the cells to simulate a random rearrangement of the fields. Second, for each place cell, the location of the peak firing rate was measured as an angle on the obroundshaped track, and peak shifts were measured as 
angular deviations between two conditions. After testing distribution uniformity (Rayleigh test), the mean direction of the shift distribution was compared to zero using a $\mathrm{V}$ test.

\section{Theta power, frequency and cycles}

Power spectra and spectrograms were computed from the detrended LFP signal using multitaper estimation methods (http://chronux.org). Power spectra were normalized for each rat as $\left(S_{i j}-\mu_{j}\right) / \sigma_{j}$, where $S_{i j}$ is the power spectrum for a given condition $i$ of rat $j$, and $\mu_{j}$ and $\sigma_{j}$ are the mean and the standard deviation of the power spectra for all of the conditions for rat $j$. Theta power was measured as the maximum power in the theta band $(6-10 \mathrm{~Hz})$ of the normalized power spectrum, and frequency was measured as the mode located in the same range (fig. 2, A and B). Theta cycles started at the peak of the LFP filtered in the theta band. Cycles shorter than $80 \mathrm{~ms}$ or longer than $200 \mathrm{~ms}$ were discarded, and only periods containing at least three contiguous cycles were selected for further analysis.

\section{Theta asymmetry and phase precession}

To preserve the asymmetrical shape of the theta oscillation, precise peak and trough times were detected on the LFP filtered between 1 and $40 \mathrm{~Hz}$. This was used to assess theta asymmetry and spike phase precession. Theta asymmetry was measured as $\log \left(\Delta t_{2} / \Delta t_{1}\right)$, where $\Delta t_{1}$ is the duration between cycle start and trough, and $\Delta t_{2}$ the duration between trough and cycle end (42). Hence, a positive value indicates a shorter descending phase, a negative value indicates a shorter ascending phase, and zero indicates symmetrical cycles (fig. 2B).

To analyze theta phase precession, in-field spike theta phases were plotted against linearized positions on the track. Slopes and significance were determined by linear-circular regression (43). Average phase precession density plots were constructed by normalizing the position in the firing field and shifting the mean spike theta phase to $180^{\circ}$, then normalizing by the total number of spikes, and averaging over cells (fig. 2E).

\section{Theta burst frequency}

Spike times were converted to unwrapped theta phases and divided by $2 \pi$, yielding units of theta cycles. Spectrograms computed on the resulting spike trains measured frequencies relative to theta, compensating for moment-to-moment variations in theta frequency over the course of a given experiment (fig. 2C,D and fig. S4A).

\section{Candidate replay events}

Candidate replay events were detected using place cell ensemble activity during slow wave sleep (SWS). SWS periods were first determined by k-means clustering of the theta $(6-10 \mathrm{~Hz}) /$ delta $(1-4 \mathrm{~Hz}) \mathrm{ra}-$ tio computed from spectrograms during sleep sessions (min duration of SWS epoch $>120$ s, permitting brief discontinuities $<1 \mathrm{~s}$; Fig. S11A). The method was validated by comparing the outcome to episodes where the animal was immobile (linear velocity $<0.05 \mathrm{~cm} / \mathrm{s}$ for at least $120 \mathrm{~s}$, with brief movements $<1 \mathrm{~s}$ ) and by visual examination of the video recordings. Candidate replay events were defined as epochs of elevated place cell spiking activity (30), i.e. when the instantaneous firing rate (1-ms bins, smoothed using a Gaussian kernel of s.d. $=10 \mathrm{~ms}$ ) reached a peak $>3$ s.d. and remained greater than the mean (fig. S11C). Candidate events lasting $>500 \mathrm{~ms}$ were excluded.

\section{Bayesian trajectory decoding}

To decode potential trajectories from place cell ensemble activity during both travel and sleep conditions, we used a previously described Bayesian position reconstruction approach (29). Briefly, in the training step, we estimated the probability $P(x)$ that the rat visited location $x$ as the normalized occupancy curve, and the average firing rate probability $\lambda_{i}(x)$ for each place cell $i$ at position $x$ as the normalized firing curve of the cell. Then in the test step, given the instantaneous firing rate vector $n$ in each phase or time window $\tau(\tau=\pi / 3$ for theta sequences, $\tau=20 \mathrm{~ms}$ for replay events), we estimated the probability $P(x \mid n)$ (fig. $3 \mathrm{~A}$ and fig. $4 \mathrm{~A}$ ):

$$
P(x \mid n)=\frac{P(n \mid x) \cdot P(x)}{P(n)}
$$

where

$$
P(n \mid x)=\prod_{i=1}^{N} \frac{\left(\lambda_{i}(x) \cdot \tau\right)^{n_{i}}}{n_{i} !} e^{-\lambda_{i}(x) \cdot \tau}
$$

assuming that the $N$ cells fired as independent Poisson processes. We only considered trajectory events involving at least three units in candidate events lasting $>60 \mathrm{~ms}$.

To compute the average reconstructed trajectory over a given condition, reconstructions from individual theta subcycles (i.e. phase windows) were cen- 
tered on the current position of the animal and averaged over successive cycles (21). This yielded an average estimate of theta sequences (fig. 3B, fig. S5A, fig. S7A, fig. S8A, fig. S9A, and fig. S10A).

To allow comparison of trajectories across conditions, the Bayesian decoding algorithm was independently trained on each of the travel sessions (e.g. firing fields in Passive 1) and applied to the same travel session (theta sequences in Passive 1) and subsequent sleep session (replay events in sleep following Passive 1). One caveat was therefore that differences in training conditions could bias the comparisons of reconstruction quality. To control for this possibility, we used four different controls:

(i) Control for cell count (fig. S7). Passive 1 typically included fewer cells. To control for this, analyses were restricted to matching random subsets of place cells in Active and Passive 2. This was repeated 30 times in order to obtain a representative data set, and up to 300 theta cycles were reconstructed for a given cell subset.

(ii) Control for spike count (fig. S8). Firing rates in Passive 1 were somewhat lower than in Active. For each rat, we randomly downsampled the spike trains of the most active place cells in Active and Passive 2 to match the median of the firing rate distribution in Passive 1.

(iii) Control for decoding quality (fig. S9). To match decoding quality across conditions, reconstruction errors were first computed in 500ms time bins for each travel condition. For Passive 1 and Passive 2, bins with a high decoding error were progressively excluded, until the median of the error distribution was smaller than the median of the error distribution in Active. Finally, positions were decoded using only theta cycles falling in these low error bins. Note that this also controlled for potential alterations in spatial coding, both at the single cell and at the population level (i.e. including higher order alterations that would not be manifested at the single cell level).

(iv) Control for field variability (fig. S10). To rule out a possible effect of firing variability between successive laps (e.g. small changes in field locations), analyses were repeated on a lap-by-lap basis, whereby the Bayesian decoding algorithm was retrained on each individual lap before sequences were assessed.
Together, these four controls ruled out the possibility that our main conclusions based on direct comparisons could be accounted for by differences in training conditions.

\section{Assessing trajectories: trajectory scores and slopes, quadrant scores}

To identify trajectories, we measured the previously defined trajectory score and slope (30) of each candidate event. These assess whether events consist of linearly aligned positions, i.e. whether the successive reconstructed positions are tightly arranged (high score) along an oblique line (high slope). Briefly, each candidate trajectory consisted of reconstructed positions $P(x \mid n)$ during $m$ successive time or phase intervals $\Delta \xi(\Delta \xi=\pi / 3$ for theta sequences, $\Delta \xi=20 \mathrm{~ms}$ for replay events). For a given candidate trajectory, the average likelihood $R$ that the rat is located within a distance $d$ of a linear trajectory defined by its velocity $v$ and starting location $\rho$ is:

$$
R(v, \rho)=\frac{1}{m} \sum_{k=0}^{m-1} P(\mid \text { pos }-(\rho+v . k . \Delta \xi) \mid \leqslant d)
$$

where the value of $d$ was set to $45 \mathrm{~cm}$ to allow for small local variations in velocity (i.e. $1 / 3$ of the mean field size, consistent with previous studies). Because in the present experiments the track was obround, for those time bins $k$ when a trajectory would specify a location beyond the end of the linearized track, the trajectory was wrapped around and continued from the start. To find the best fit line (i.e. maximize $R$ ), we evaluated all possible combinations of $v$ and $\rho$ that yielded a total distance less than the track length $(6 \mathrm{~m})$.

Thus, genuine trajectories would yield both high scores and slopes $(R \gg 0,|v| \gg 0)$, whereas static representations of current position would result in high scores but low or zero slopes $(R \gg 0, v \sim 0)$, and random activity would be associated with low scores $(R \sim 0)$, but possibly spuriously high slopes.

In order to assess the significance of trajectory scores, we used a shuffling procedure developed by (30). Briefly, we generated $n=5,000$ shuffled candidates by shifting each reconstructed position $P(x \mid n)$ by a random distance, yielding a null distribution of scores. Note that this selectively scrambled the linear arrangement of reconstructed positions, while preserving the spatial coherence of positions represented by individual cell assemblies (contrary to e.g. independent shuffling of individual spike trains). In other words, shuffling was applied 
at the level of cell assemblies rather than single neurons.

More precisely, shuffles were generated by circularly shifting the decoded probabilities within each phase or time window by a random number of bins. The Monte Carlo $p$-value was calculated as the proportion of scores in the shuffled distribution greater than the score of the candidate trajectory. Candidate trajectories with a $p$-value $\leqslant 0.05$ were considered significant. In addition, in order to compare trajectory events from different rats recorded in different conditions (i.e. with differences in number of neurons, decoding quality, etc.), trajectory scores were normalized by computing a non-parametric equivalent of a z-score, i.e. by subtracting the mean and dividing by the standard deviation of the distribution of shuffled scores (fig. 3C, fig. S7C, fig. S8C, fig. S9C, and fig. S10C). In Figure 4B, to assess changes in sleep activity induced by preceding awake behavior, for each rat, we evaluated each score in post-sleep relative to baseline sleep by computing a non-parametric equivalent of a z-score, i.e. by subtracting the median score in baseline sleep, and dividing by the difference between the first and third quartiles of the distribution of scores in baseline sleep. These scores relative to baseline were then pooled across rats and compared between conditions. Candidate replay events during baseline sleep were decoded using the Bayesian algorithm trained on the same travel condition as the post sleep session.

To compute the proportion of significant theta sequences (fig. 3C), only events with significant score and positive slope were counted (the same conclusion was reached without the slope restriction: $14.66 \%$ in Passive 1, 20.20\% in Active, $16.37 \%$ in Passive 2; all proportions were significantly greater than shuffled control proportions and all proportions were different between one another; data not shown).

To confirm the above trajectory assessment using an independent measure, we used the quadrant score first described in (27). Similar to the trajectory score and slope method, each candidate trajectory was first described by a probability matrix consisting of successive reconstructed positions $P(x \mid n)$. The central zone of this probability matrix was defined as $\pm 1.5 \mathrm{~m}$ from the current position of the animal and $\pm 2 / 3$ theta cycle around the cycle trough. This was divided equally into four quadrants. The summed decoded probabilities in the top left and bottom right quadrants (oppo- site to the current running direction of the animal) were subtracted from the sum in the bottom left and top right quadrants (along the current running direction), then normalized by the sum of all four quadrants (fig. 3F, fig. S7D, fig. S8D, fig. S9D, and fig. S10D). Hence, positive differences would correspond to theta sequences sweeping in the running direction of the animal, whereas differences close to zero would indicate a lack of sequential structure in the decoded probabilities.

\section{Assessing trajectories: Z-proportions and proportion ratios}

In order to simultaneously visualize the distribution of trajectory scores and slopes across candidate trajectories and contrast them between behavioral conditions, events were first sorted according to their trajectory scores and slopes. Each cell of the resulting score-slope matrix contains the number of events within that specific range of scores and slopes divided by the total number of events. To assess the statistical significance of these proportions, we created $n=5,000$ surrogate score-slope matrices using shuffled data as described in the previous section. We then z-scored the proportion in each cell of the observed matrix (data) relative to the mean and standard deviation of the 5,000 proportions located in the same cell of the surrogate matrices (z-proportion; fig. 3E, fig. 4D, fig. S7E, fig. S8E, fig. S9E, fig. S10E, fig. S12F, and fig. S13C).

A complementary approach was used in Figure S13D, where the color code indicates the proportion ratio $r$ defined as:

$$
r_{c}=\frac{1}{m} \sum_{i=1}^{m} \frac{n_{\text {data }}}{n_{\text {data }}+n_{\text {shuffle }_{i}}}
$$

where $m$ is the number of shuffles, $n_{\text {data }}$ the number of events in cell $c$ of the score-slope matrix, and $n_{\text {shuffle }_{i}}$ the number of events in cell $c$ for the $i$-th shuffle. Thus, a ratio of 0.5 indicates that the cell contains the same proportion of observed and shuffled data, ratios above 0.5 indicate that scores and slopes in cell $c$ are enriched in the data relative to the shuffle (up to a maximum ratio of 1 indicating that events with the corresponding score and slope were observed in the data but in none of the 5,000 shuffle iterations), and ratios below 0.5 indicate that the scores and slopes in $c$ are depleted in the data relative to the shuffle (down to a minimum ratio of 0 indicating that events with the corresponding score and slope occurred in the shuffle but not in the original data). 


\section{Pairwise bias correlations}

In order to directly compare the order between any two spike sequences, we used the pairwise bias correlation method developed by (44). Briefly, we computed a bias matrix $B_{k}$ for each sequence $k$ :

$$
B_{k}(i, j)=\frac{n_{k}(i, j)-n_{k}(j, i)}{n_{k}(i) \cdot n_{k}(j)}
$$

where $n_{k}(i)$ is the number of spikes emitted by neuron $i$ in the $k$-th sequence, and $n_{k}(i, j)$ is the number of times neuron $i$ spiked before neuron $j$ in the $k$-th sequence. $B_{k}(i, j)$ therefore reflects the bias of $i$ to spike before $j$ in the $k$-th sequence, taking values between -1 ( $i$ never precedes $j$ ) and 1 ( $i$ always precedes $j$ ), with 0 representing no bias ( $i$ precedes $j$ half of the time).

The correlation between two sequences $k$ and $l$ is $\rho(k, l)=\cos \theta$, where $\theta$ is the angle between the directions of $B_{k}$ and $B_{l}$, when $B_{k}$ and $B_{l}$ are considered as vectors. Therefore

$$
\rho(k, l)=\frac{\sum_{i, j} B_{k}(i, j) \cdot B_{l}(i, j)}{\sqrt{\sum_{i, j} B_{k}(i, j)^{2}} \cdot \sqrt{\sum_{i, j} B_{l}(i, j)^{2}}}
$$

where $i$ and $j$ are neurons that fired in both sequences $k$ and $l$.

Comparisons within and between theta sequences and replay events were restricted to sequences including three or more (common) neurons. Autocorrelations $(k=l)$ were discarded from the analysis.
To assess significance for the correlation of a given pair $(k, l)$ of sequences, we constructed a surrogate distribution of pairwise bias correlations by shuffling the spike order in sequence $l$. The observed pairwise bias correlation $\rho(k, l)$ was deemed significant if it exceeded $95 \%$ of the shuffled distribution.

In Figures 3G and 4G, each condition (baseline sleep, Passive 1, etc.) was subdivided into 20 temporal bins. To represent the similarity between two given temporal bins, we first computed for each recording session the median correlation between individual sequences within these bins, then took the median across all sessions.

\section{Ripple detection}

LFPs from all channels located in or close to the pyramidal layer were detrended and filtered between 100 and $250 \mathrm{~Hz}$, then averaged. In order to prevent spurious detection due to high frequency noise contamination, the detrended signal from the selected channels was also filtered between 300 and $500 \mathrm{~Hz}$, then averaged and subtracted from the mean filtered signal. Negative values, reflecting high frequency noise periods, were discarded while all positive values were z-scored, and ripples were defined as epochs during which the values remained above 1 s.d. with a peak greater than 3 s.d. Excessively brief $(\leqslant 20 \mathrm{~ms})$ or long $(\geqslant 110 \mathrm{~ms})$ epochs were excluded from subsequent analyses. 
A
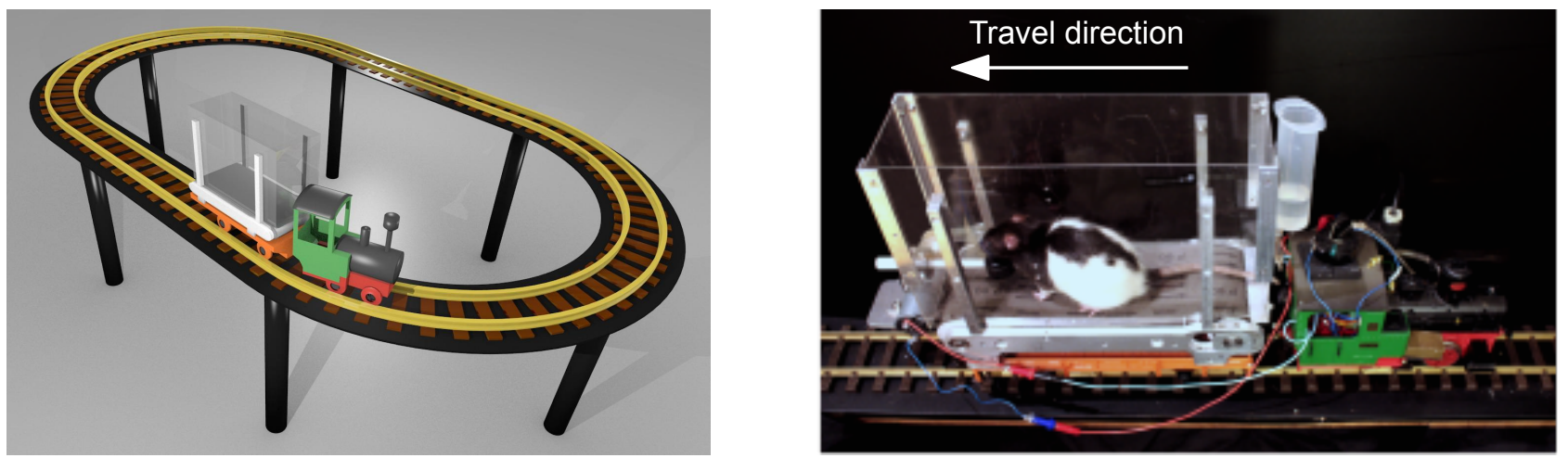

B

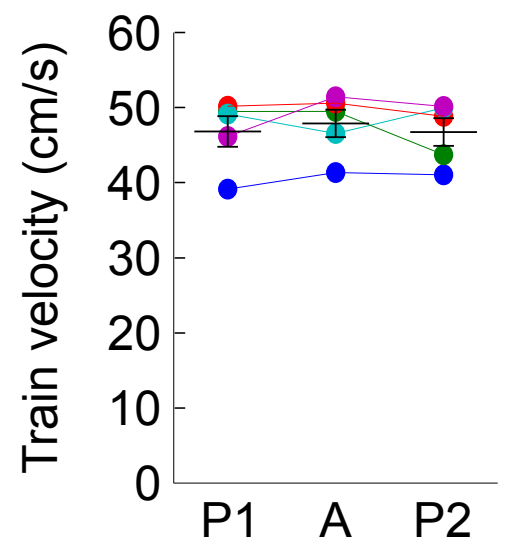

C

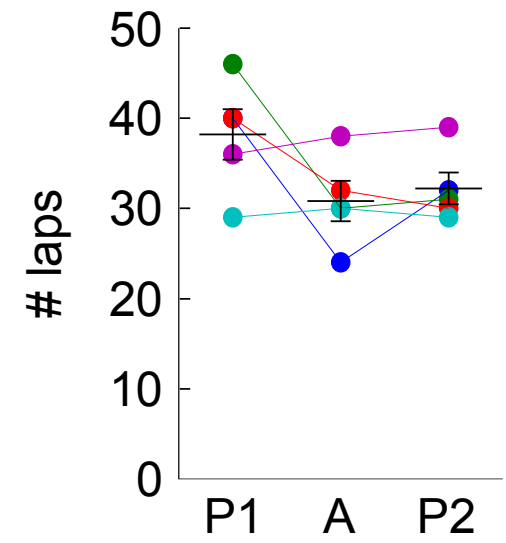

D

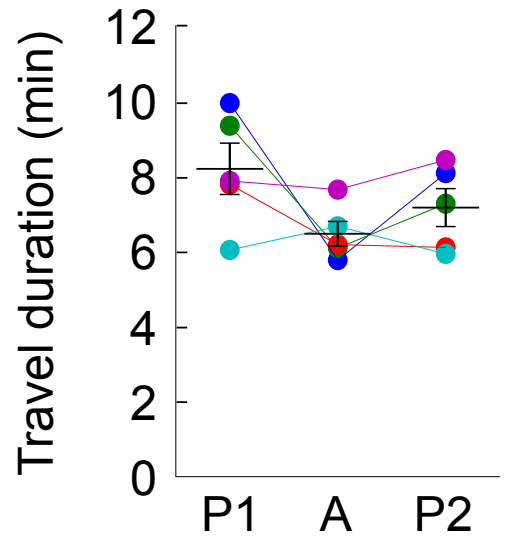

Fig. S1. Behavioral task.(A) Left, 3D representation of the experimental setup. Right, rats were transported on a miniature treadmill in a model train. Rats were displaced in the forward direction at all times. The locomotive was placed at the rear of the wagon in order to avoid obstruction of the visual field of the animal. Rats traveled in the novel environment while either running on the treadmill (Active travel) or standing still (Passive travel). (B) Train velocity in each condition for each rat $\left(n=5\right.$ rats, mean \pm s.e.m., P1: $46.80 \pm 2.04 \mathrm{~cm} . \mathrm{s}^{-1}, \mathrm{~A}: 47.87 \pm 1.83 \mathrm{~cm} . \mathrm{s}^{-1}$, P2: $46.73 \pm 1.85 \mathrm{~cm} . \mathrm{s}^{-1}$; repeated measures ANOVA, $\left.P>0.05\right)$. (C) Number of laps traveled by each rat in each condition ( $n=5$ rats, mean \pm s.e.m., P1: $38.2 \pm 2.8$ laps, A: $30.8 \pm 2.24$ laps, P2: $32.2 \pm 1.77$ laps; repeated measures ANOVA, $P>0.05)$. (D) Travel duration for each rat in each condition $(n=5$ rats, mean \pm s.e.m., P1: $8.24 \pm 0.69 \mathrm{~min}, \mathrm{~A}: 6.50 \pm 0.33 \mathrm{~min}, \mathrm{P} 2: 7.20 \pm 0.51 \mathrm{~min}$; repeated measures ANOVA, $P>0.05)$. 
A

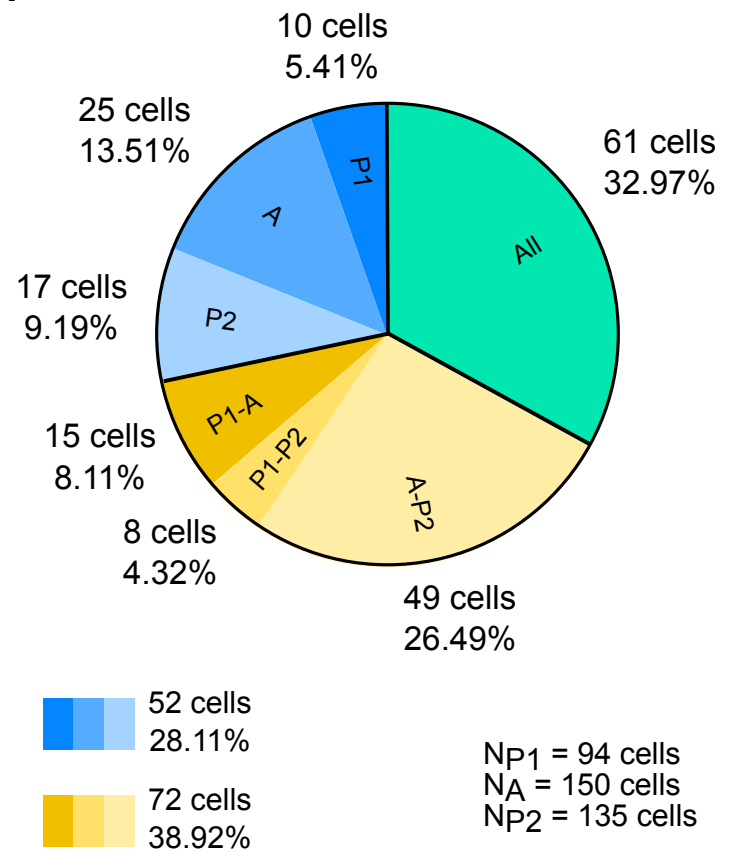

B

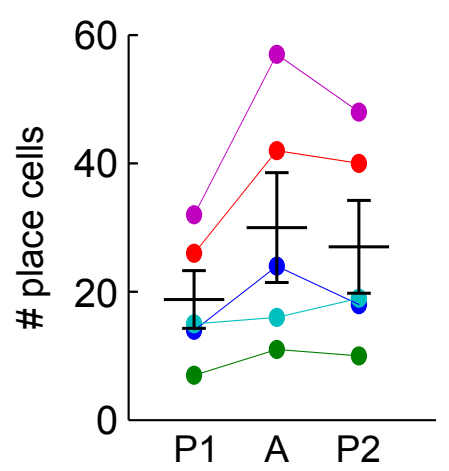

C

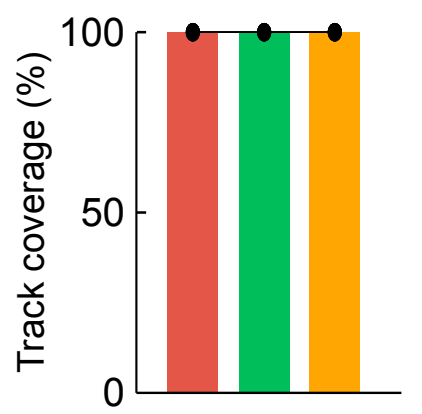

D
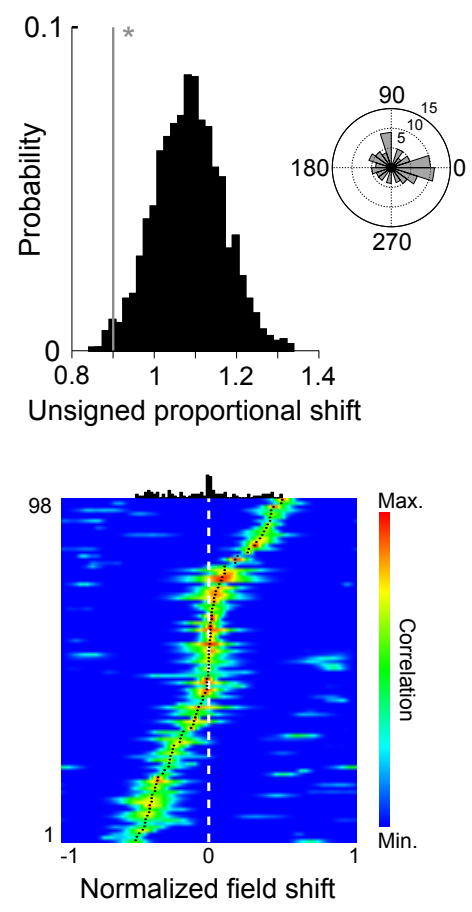

Fig. S2. Place cell activity during travels. (A) Number of individual place cells recorded in one or more travel sessions. Place cells recorded in only one or two conditions are represented in blue and yellow, respectively. (B) Total number of place cells recorded in each travel condition (repeated measures ANOVA, ${ }^{*} P<0.05$ ). (C) Percentage of the track covered by place fields in each travel condition. (D) Stability of place fields between Passive 1 and Passive 2. Top, mean unsigned proportional shift $\sigma\left({ }^{*} P<0.02\right)$. Histogram represents the distribution of $\sigma$ for $n=2,000$ bootstrap random remapping data sets. Inset, circular distribution of angular differences between in-field peak firing rates (Rayleigh test, $P>0.05$, V-test against $0,{ }^{*} P<0.05$ ). Bottom, spatial cross-correlograms of firing fields. Black dots represent correlogram modes. Top black histogram, mode distribution. 


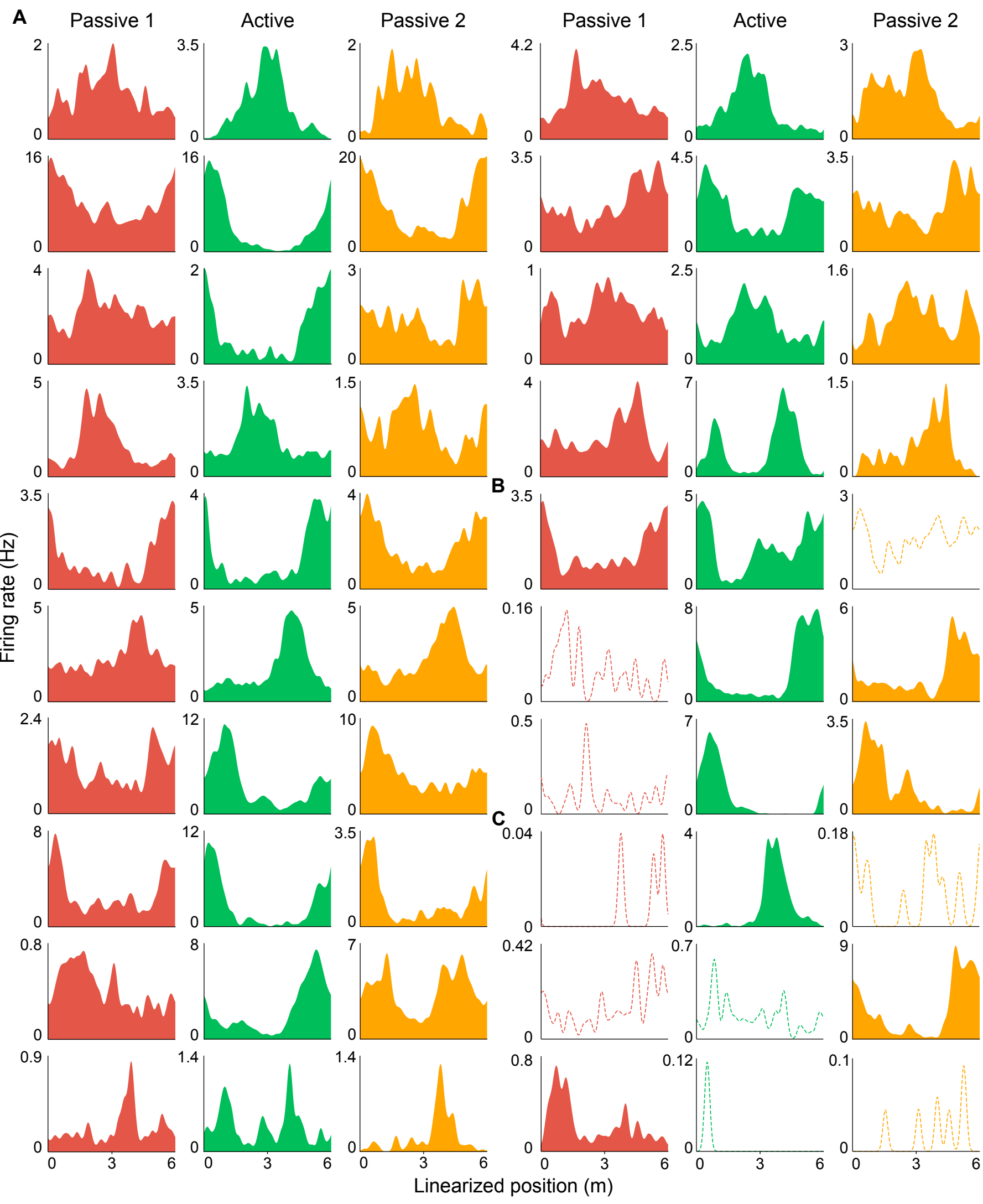

Fig. S3. Example firing fields recorded in the three travel conditions. (A-C) Firing curves of putative pyramidal cells defined as place cells (see Methods) in all three travel conditions (A), in two travel conditions (B), or in one travel condition (C). Dotted lines, firing curves of cells lacking an identified place field. 
A

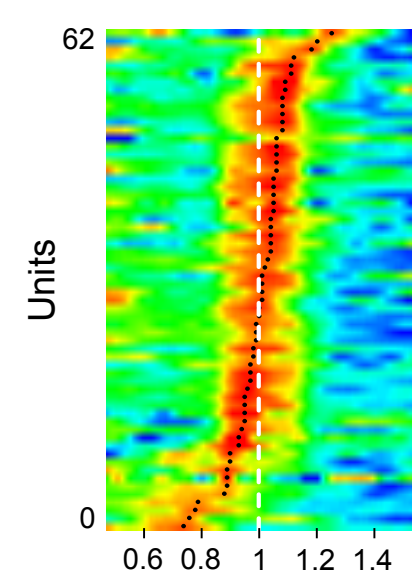

C Frequency $\left(\right.$ cycle $\left.^{-1}\right)$

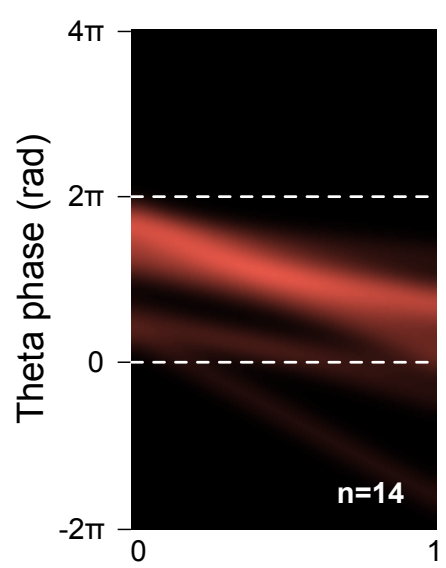

Normalized field size
Active

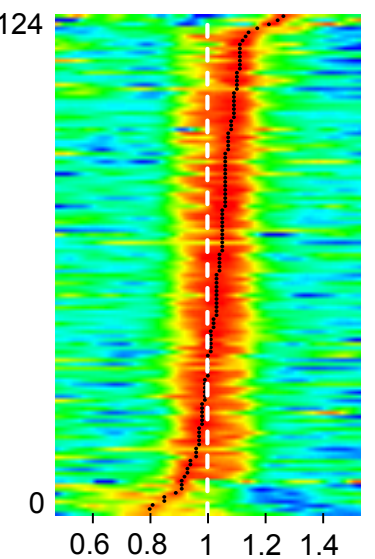

Frequency $\left(\right.$ cycle $\left.^{-1}\right)$

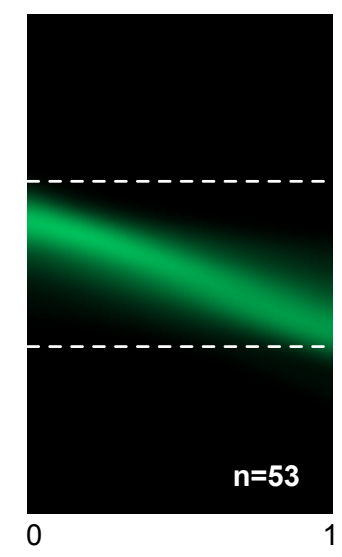

Normalized field size
Passive 2

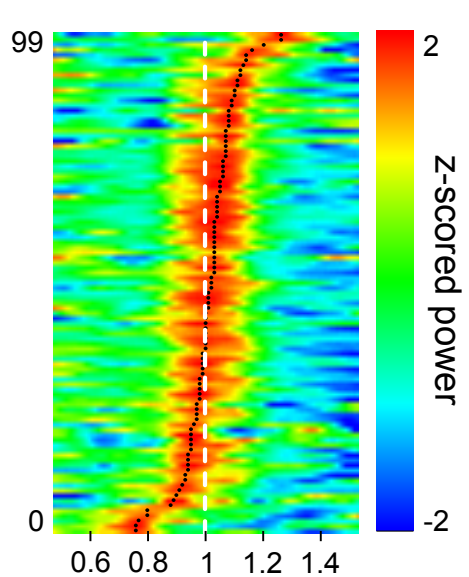

Frequency $\left(\right.$ cycle $\left.^{-1}\right)$

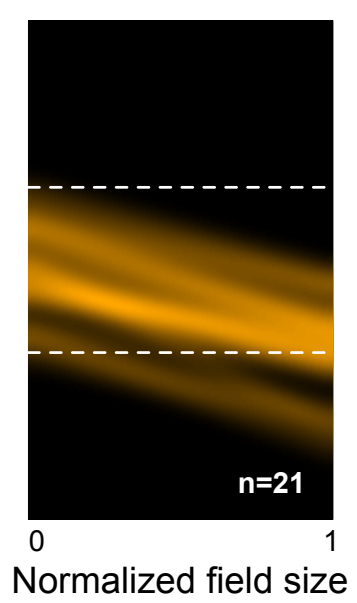

B

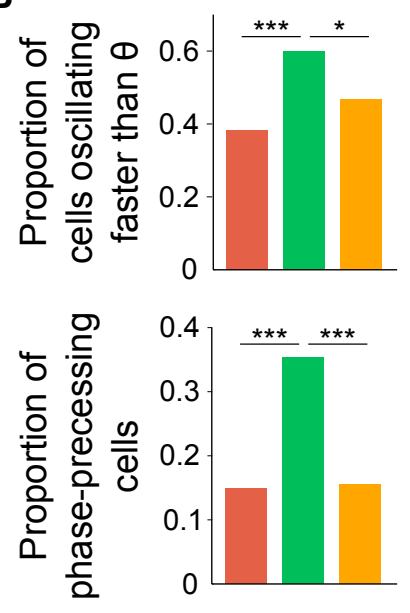

D

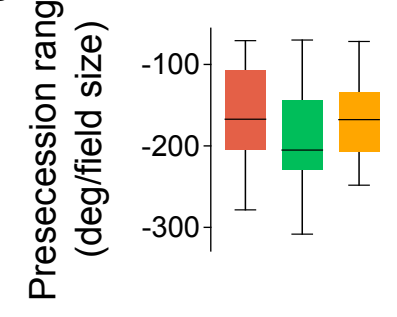

E

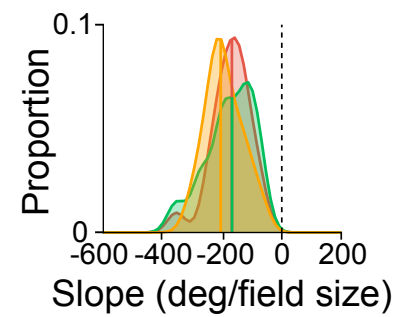

Fig. S4. Phase precession is altered during passive travel. (A) Power spectra of unit spike trains measured relative to theta (dashed white line indicates theta frequency). Only place cells with a peak around $\pm 25 \%$ of theta frequency are shown. Black dots indicate spectral modes. (B) Proportion of place cells oscillating faster than theta frequency (top, Passive 1: 36/94, Active: 90/150, Passive 2: 63/135) and proportion of significant phase-precessing cells (bottom, Passive 1: 14/94, Active: 53/150, Passive 2: 21/135).(C) Density of phase precession slopes for significant cells. (D) Range of phase precession for significant cells. Passive and Active travels were not significantly different (KW test, $P>0.05$ ). (E) Distributions of significant phase precession slopes (KS test, $P>0.05$ ). Solid lines represent medians (KW test, $P>0.05$ ). 
A
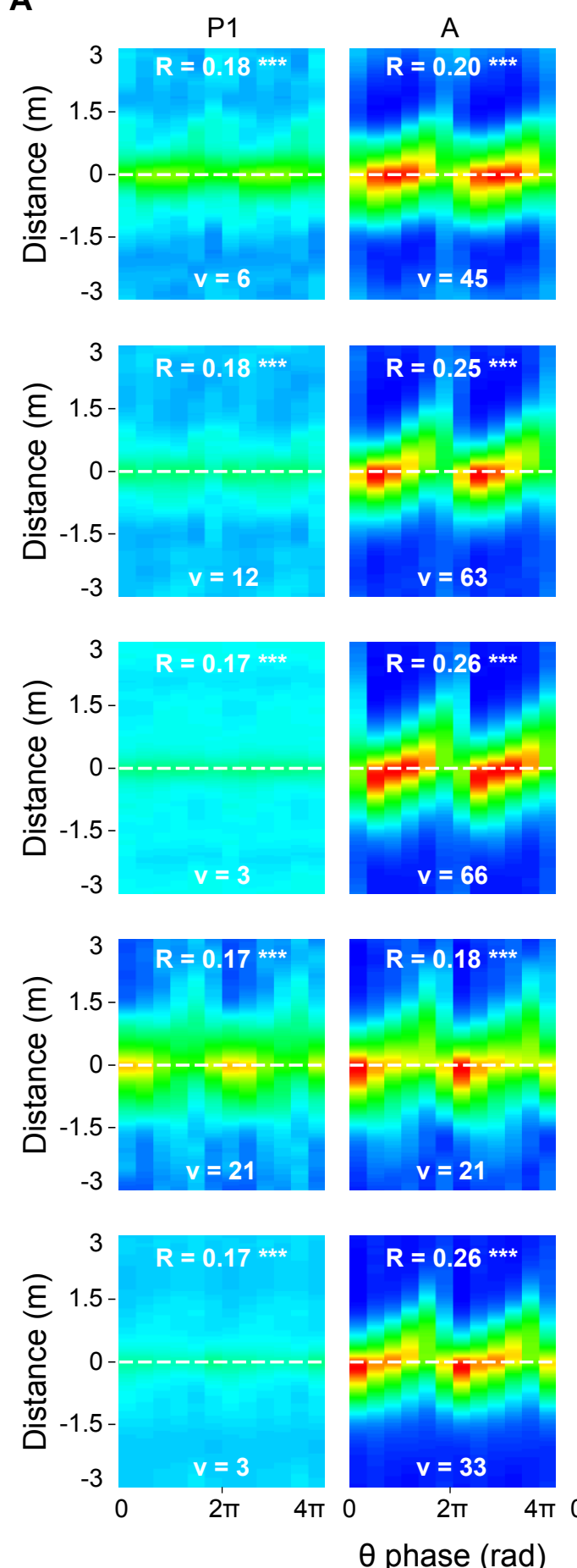

P2
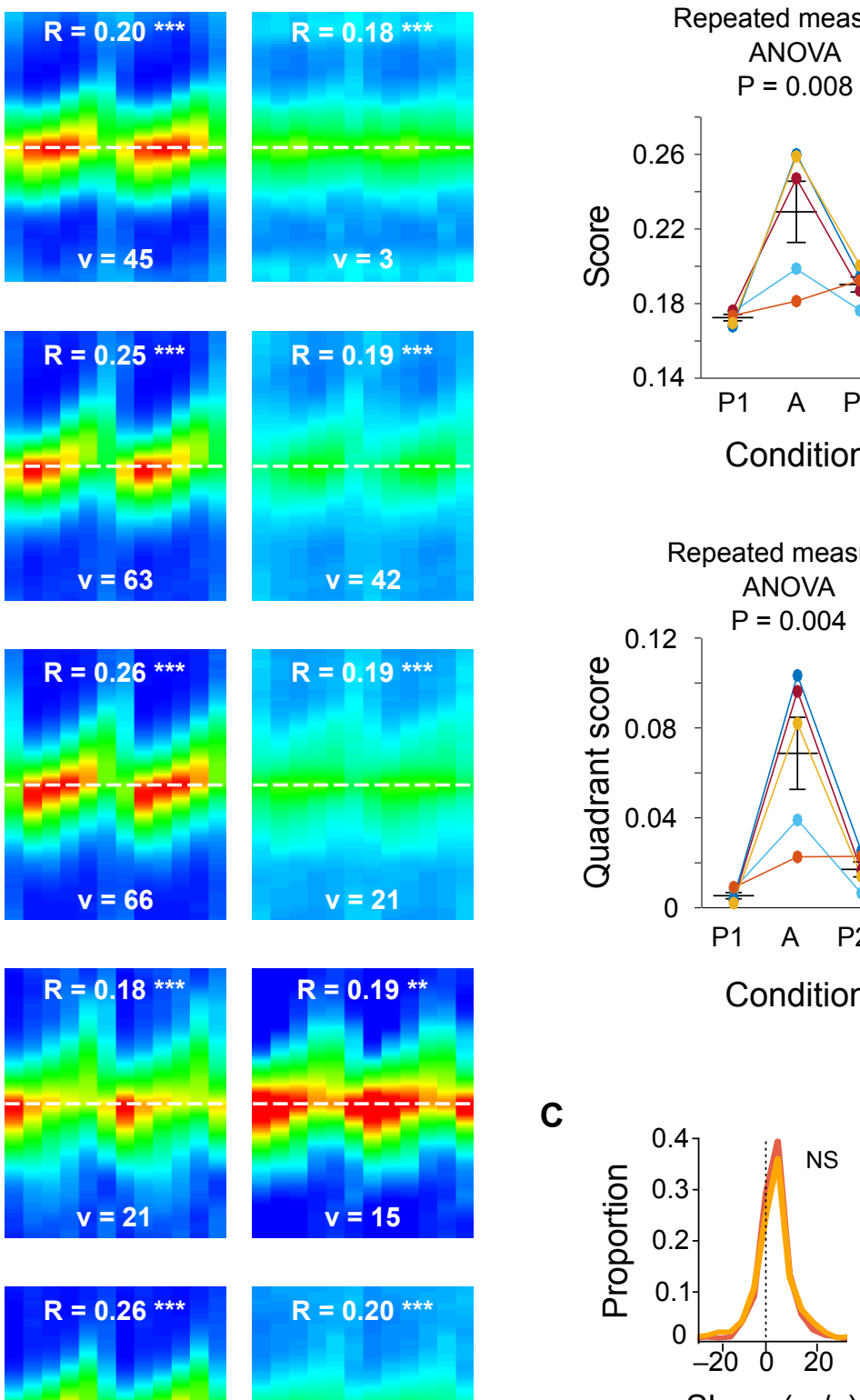

Slope $(\mathrm{m} / \mathrm{s})$

B
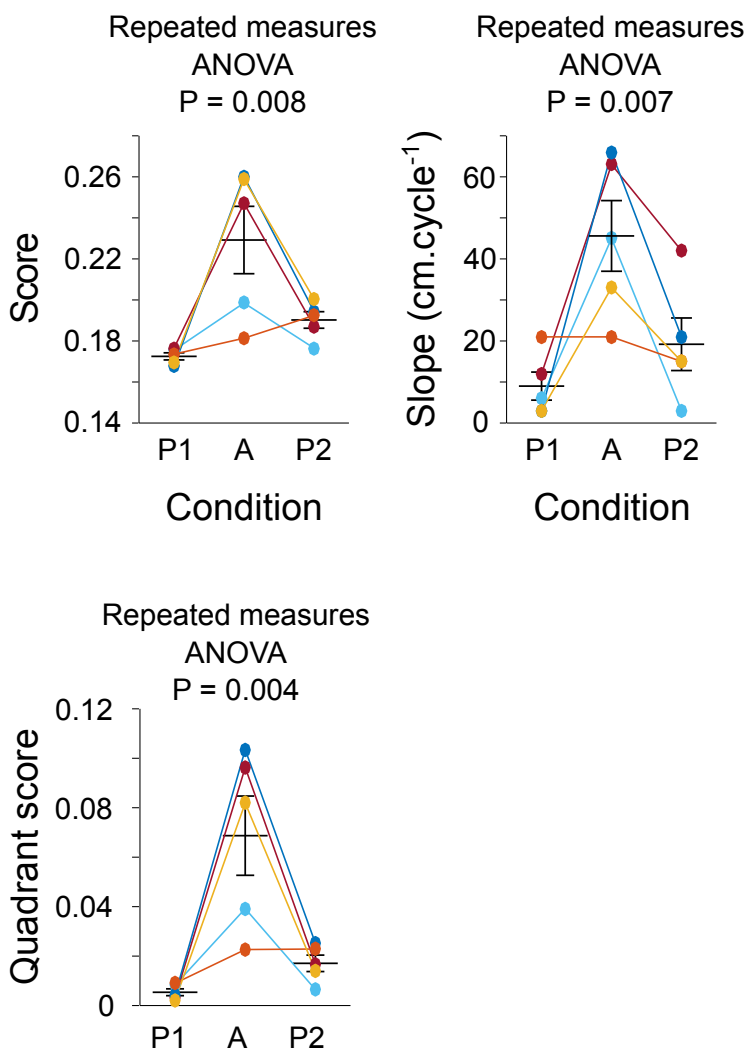

Condition

C
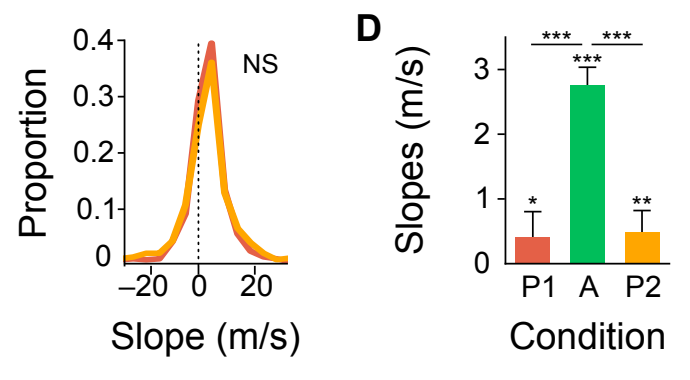

Fig. S5. Position decoding from theta-scale place cell activity for each rat. (A) Average reconstruction of rat position estimated across theta cycles for the 5 rats used in the study (dashed white line, actual animal position). Trajectory score $R$ and slope $v$ (in $\mathrm{cm} . c y c l \mathrm{e}^{-1}$ ) are indicated above each reconstruction. For each rat, the same scale was applied to the three reconstructions. (B) Trajectory scores, slopes and quadrant scores of reconstructions shown in (A) (mean \pm s.e.m.). (C) Distributions of significant theta sequence slopes in Passive 1 and Passive 2 (KS tests, P1-P2, $P>0.05$ ). (D) Significant theta sequence slopes (KW test, ${ }^{* * *} P<10^{-20}$ ). 
A

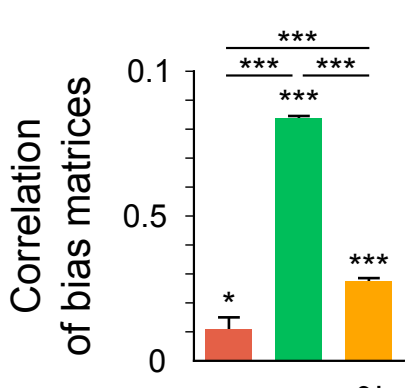

B

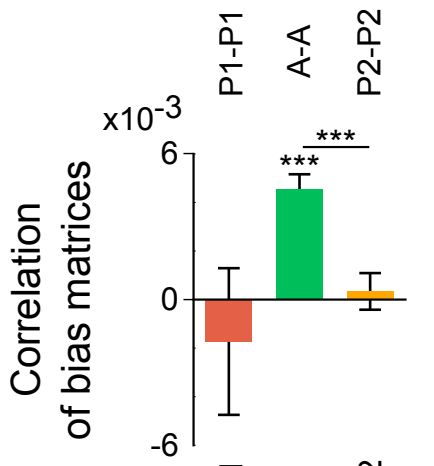

C
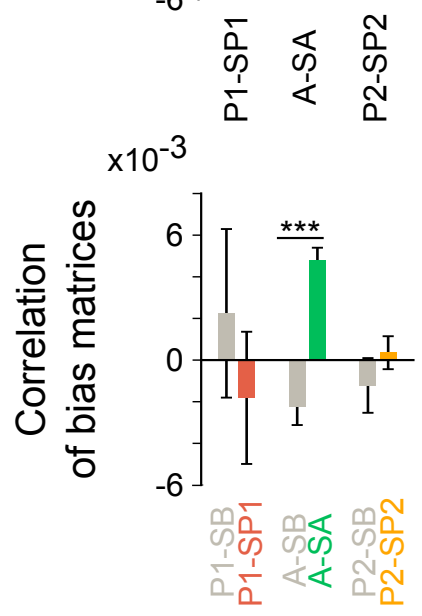

D

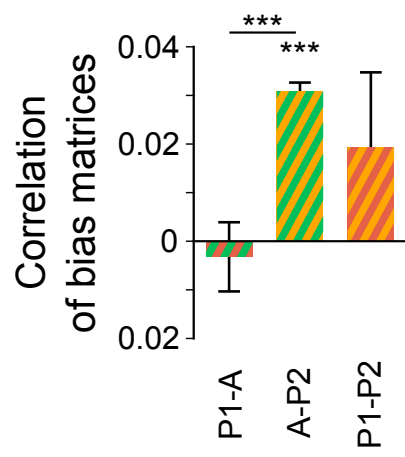

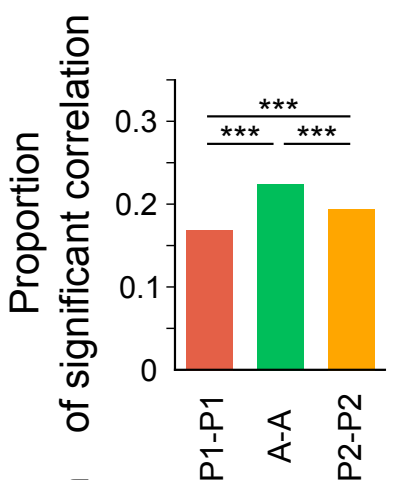
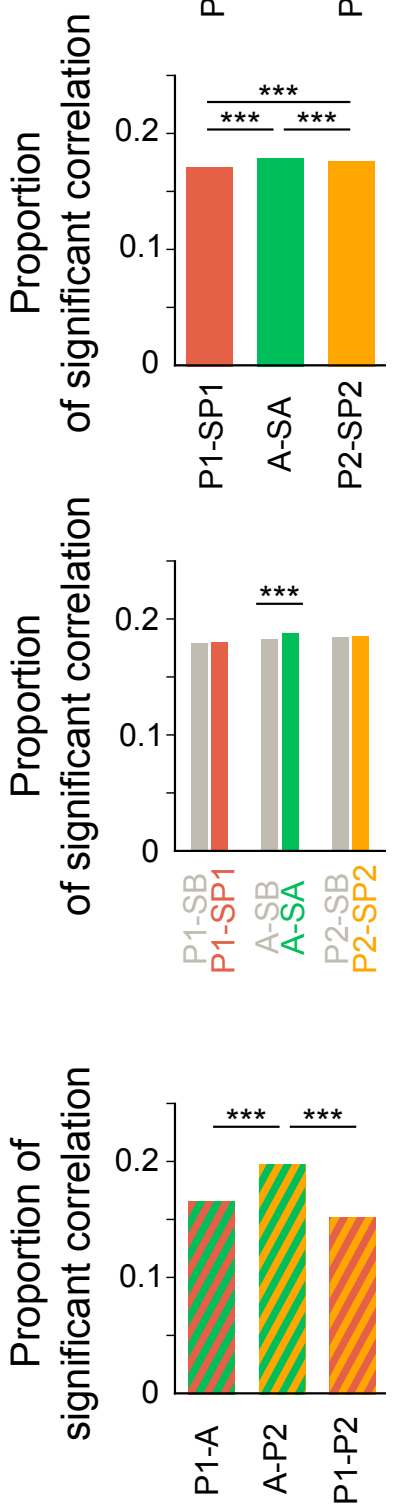

Fig. S6. Direct measure of correlations between sequences within and across conditions. (A-D) Left, average correlation between sequence biases (KW test). Right, proportion of significantly correlated pairs of sequences (binomial proportion test, ${ }^{* * *} P<0.001$ ). (A) Self-consistency within travel conditions (correlation between theta sequences within the same condition). (B) Similarity between awake (theta sequences) and respective post-sleep (candidate replay events). (C) Comparison of the similarity in (B) with the similarity between awake (theta sequences) and baseline sleep (grey). (D) Similarity of theta sequences between travel conditions. 

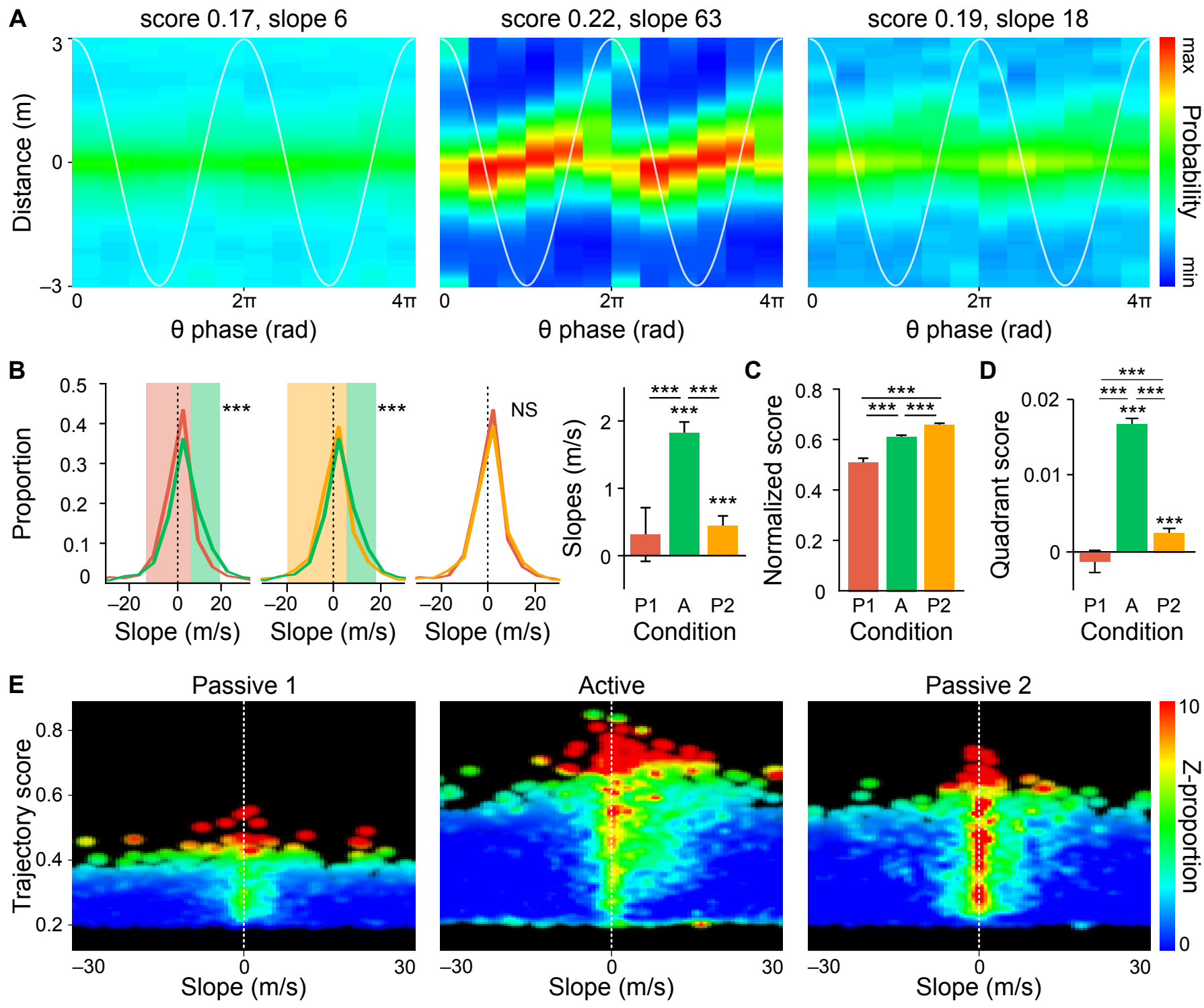

Fig. S7. Reduced cell count does not account for theta sequence degradation during passive travel. For each rat, subsets of place cells were selected in Active and Passive 2 to match the number of place cells recorded in Passive 1, and used to decode position in space. This downsampling procedure was repeated 30 times. (A) Rat position was then estimated across individual theta subcycles and averaged across all rats (same as in fig. 3B). Trajectory scores and slopes (in $\mathrm{cm} \mathrm{cycle}^{-1}$ ) are indicated above each reconstruction. (B) Distributions of significant theta sequence slopes (KS tests; left: $\mathrm{P} 1-\mathrm{A},{ }^{* * *} \mathrm{P}<10^{-13}$; center: $\mathrm{A}-\mathrm{P} 2{ }^{* * *} \mathrm{P}<10^{-43}$; right: $\mathrm{P} 1-\mathrm{P} 2, P>0.05)$. Colored bands indicate significant differences. Right, slopes of significant theta sequences (KW test, ${ }^{* * *} P<10^{-39}$ ). (C) Normalized score of theta sequences (KW test, ${ }^{* * *} P<10^{-9}$ ). (D) Quadrant score computed from individual theta cycles $\left(\mathrm{KW}\right.$ test, ${ }^{* * *} P<10^{-75}$ ). (E) Distribution of theta sequence quality assessed by joint trajectory score and slope (color codes for proportion relative to shuffled control data). 

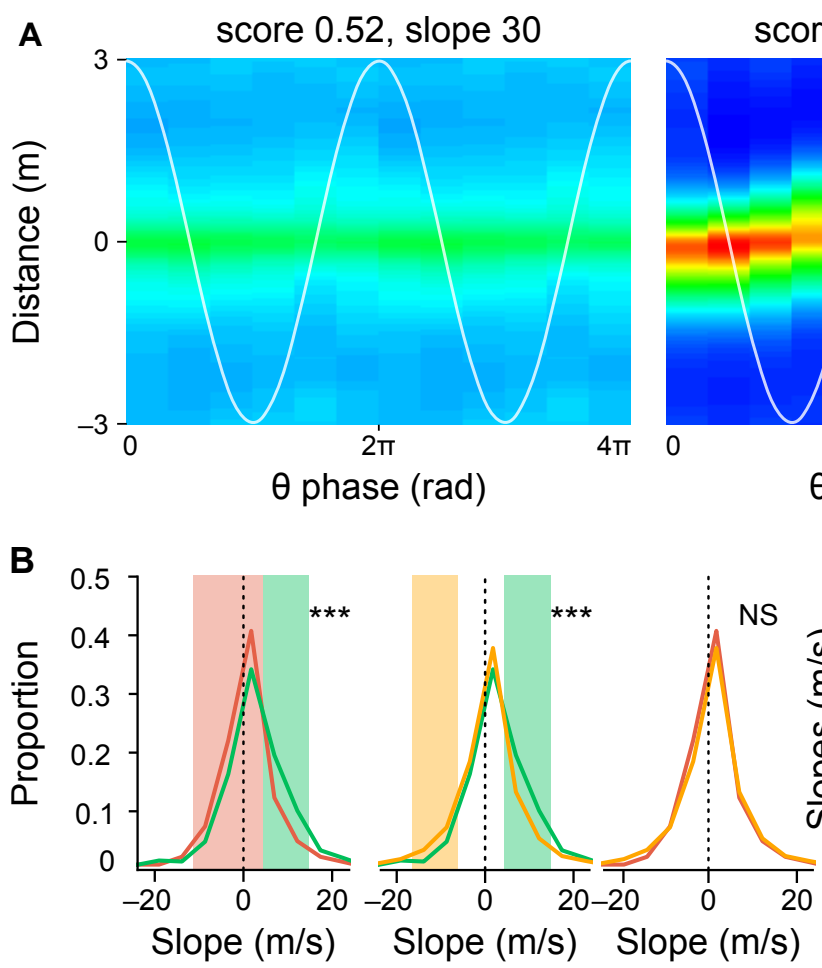

score 0.58 , slope 66

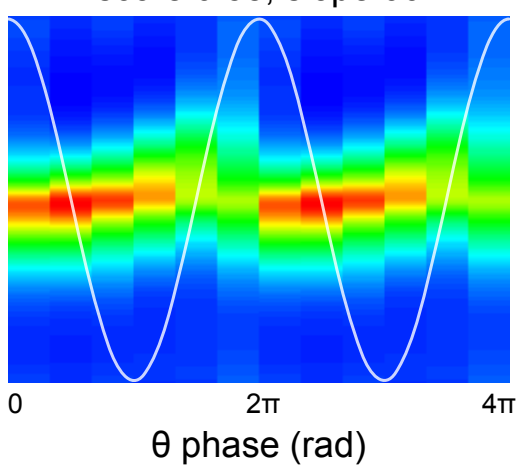

score 0.54 , slope 48

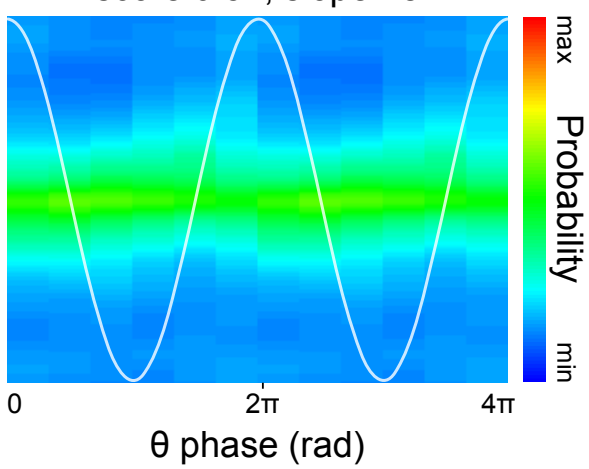

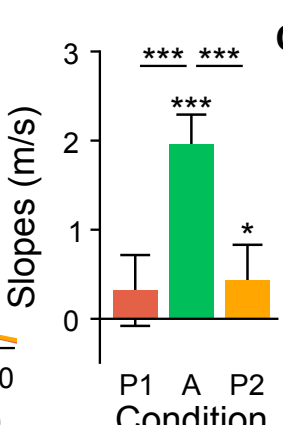

Condition

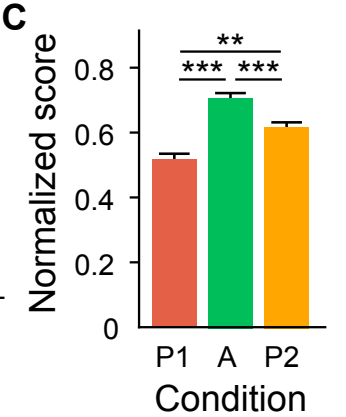

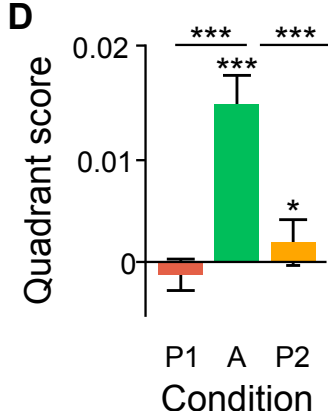

E

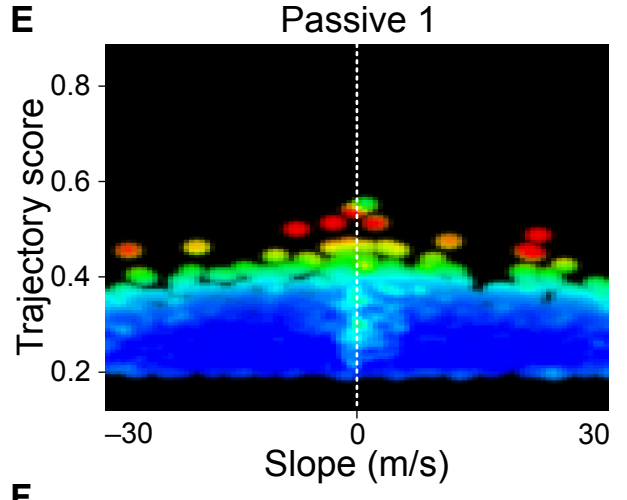

F

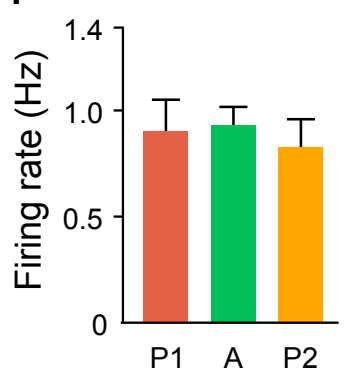

Condition

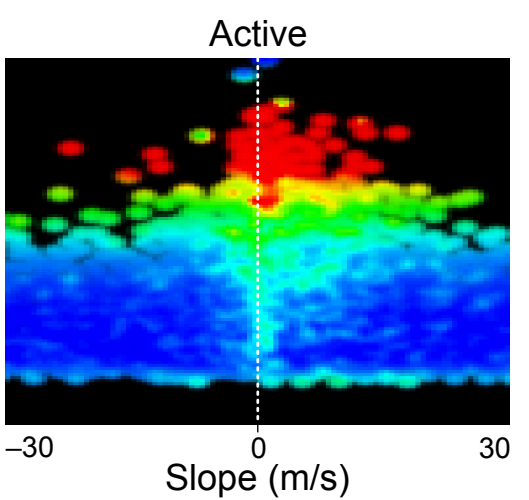

Slope $(\mathrm{m} / \mathrm{s})$

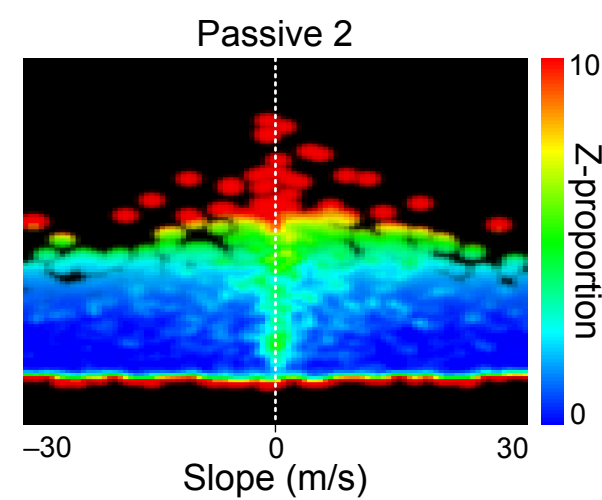

Slope $(\mathrm{m} / \mathrm{s})$

Fig. S8. Reduced firing rate does not account for theta sequence degradation during passive travel. For each rat, random spikes of place cells in Active and Passive 2 were progressively removed until the median firing rate of place cells in Active and Passive 2 was smaller than the median firing rate of Passive 1 place cells. (A) Rat position was then estimated across individual theta subcycles and averaged across all rats (same as in fig. 2B). Trajectory score and slope (in $\mathrm{cm} . \mathrm{cycle}^{-1}$ ) are indicated above each reconstruction. (B) Distributions of significant theta sequence slopes (KS tests; left: $\mathrm{P} 1-\mathrm{A},{ }^{* * *} P<10^{-9}$; center: $\mathrm{A}-\mathrm{P} 2,{ }^{* * *} P<10^{-9}$; right: $\mathrm{P} 1-\mathrm{P} 2, P>0.1)$. Colored bands indicate significant differences. Right, slopes of significant theta sequences $\left(\mathrm{KW}\right.$ test, ${ }^{* * *} P<10^{-11}$ ). (C) Normalized score of theta sequences (KW test, ${ }^{* * *} P<10^{-10}$ ). (D) Quadrant score computed from individual theta cycles (KW test, ${ }^{* * *} P<10^{-7}$ ). (E) Distribution of theta sequence quality assessed by joint trajectory score and slope (color codes for proportion relative to shuffled control data). (F) Resulting place cell firing rates following spike downsampling $(P 1: 0.905 \pm 0.145 \mathrm{~Hz}, \mathrm{~A}: 0.933 \pm 0.083 \mathrm{~Hz}, \mathrm{P} 2$ : $0.829 \pm 0.130 \mathrm{~Hz}$; KW test, $P>0.90)$. 


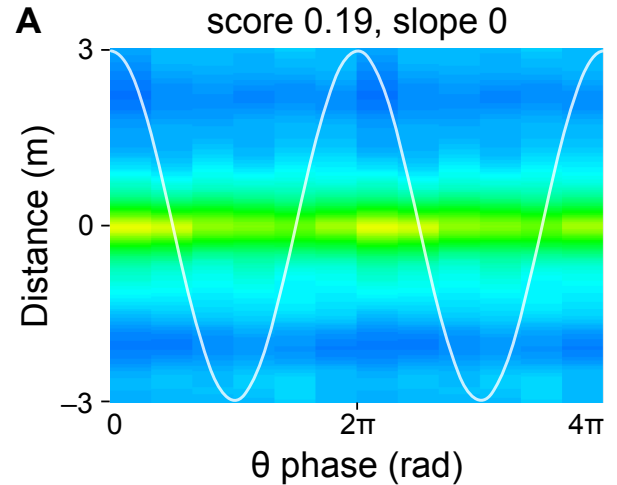

B

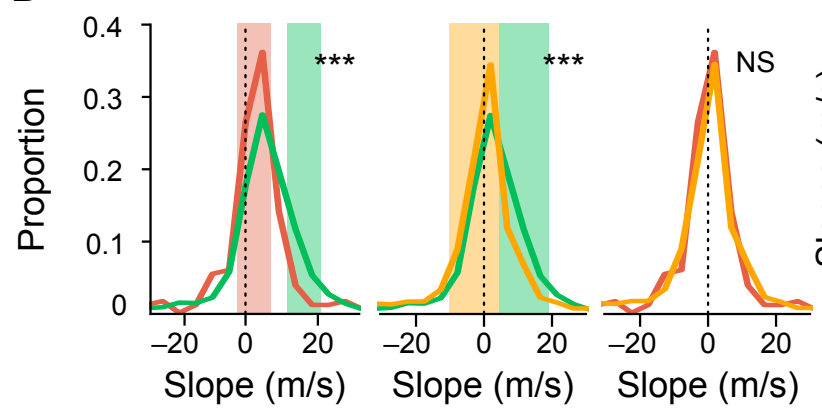

score 0.21 , slope 48

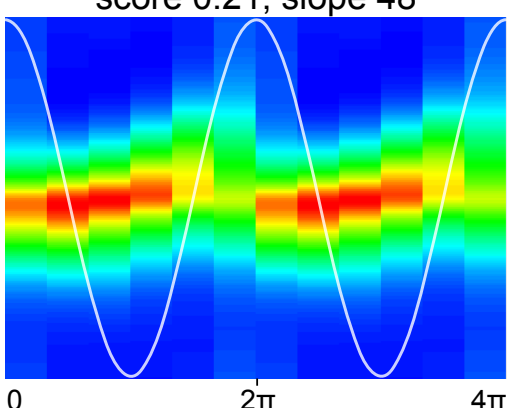

$\theta$ phase (rad)

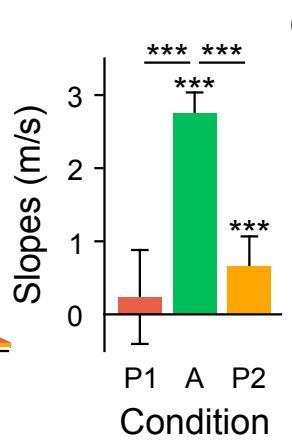

C

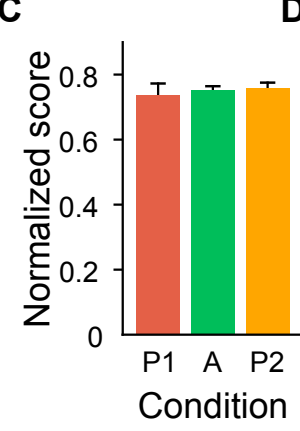

score 0.20 , slope 18

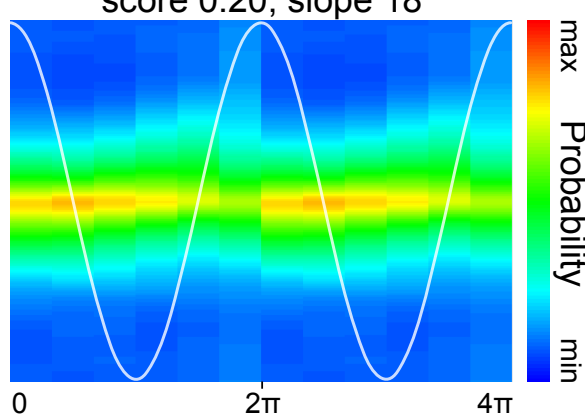

$\theta$ phase (rad)

D

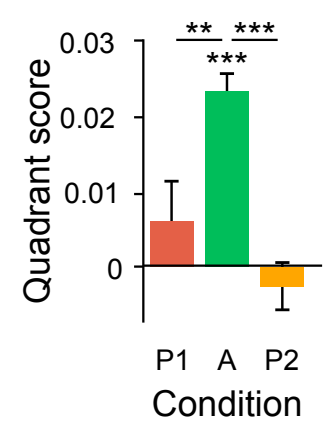

E

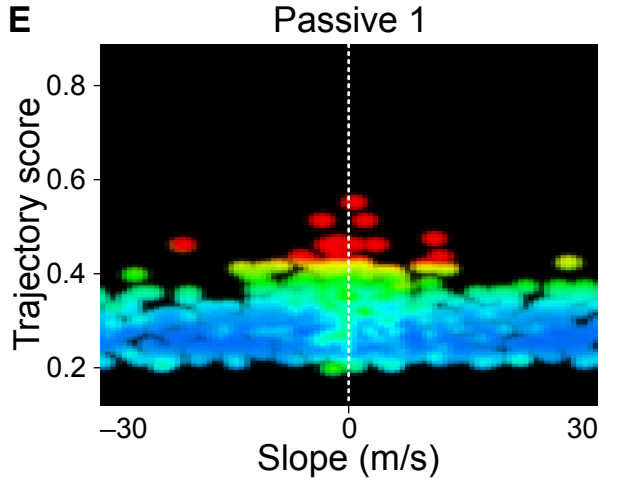

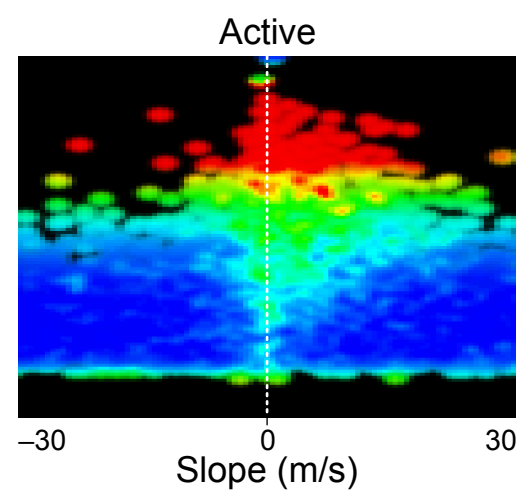

Slope $(\mathrm{m} / \mathrm{s})$

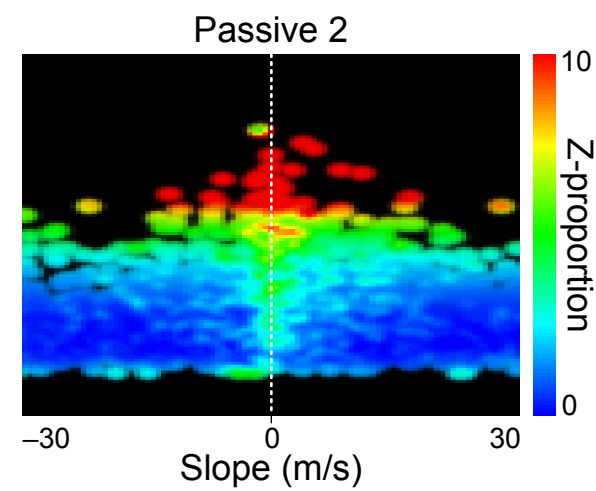

Slope $(\mathrm{m} / \mathrm{s})$

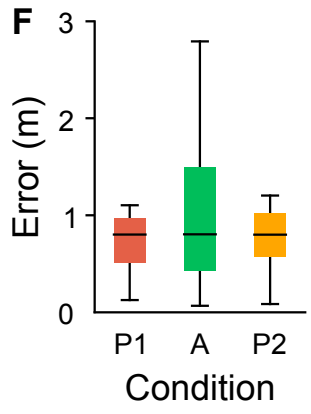

Fig. S9. Reduced decoding quality cannot account for theta sequence degradation during passive travel. In order to control for reduced decoding quality in passive travel sessions, the present analyses were restricted to theta cycles in Passive 1 and Passive 2 where decoding error (estimated in 500 ms time bins) was low, i.e. close to the error median in Active (see Methods). (A) Average Bayesian reconstruction of position (relative to actual position of the animal) across theta subcycles for all rats (as in fig. 3B). Trajectory scores and slopes (in cm.cycle ${ }^{-1}$ ) are indicated above each reconstruction. (B) Distributions of significant theta sequence slopes (KS tests; left: $\mathrm{P} 1-\mathrm{A},{ }^{* * *} P<10^{-9}$; center: A-P2, ${ }^{* * *} P<10^{-16}$; right: $\left.\mathrm{P} 1-\mathrm{P} 2, P>0.05\right)$. Colored bands indicate significant differences. Right, slopes of significant theta sequences (KW test, ${ }^{* * *} P<10^{-15}$ ). (C) Normalized score of theta sequences (KW test, $P>0.05$ ). (D) Quadrant score computed from individual theta cycles (KW test, ${ }^{* * *} P<10^{-16}$ ). (E) Distribution of theta sequence quality assessed by joint trajectory score and slope (color codes for proportion relative to shuffled control data). (F) Decoding errors were similar in all travel conditions following the restriction procedure described here (P1: $0.802 \pm 0.018, A: 0.80 \pm 0.023, P 2: 0.802 \pm 0.014)$. 

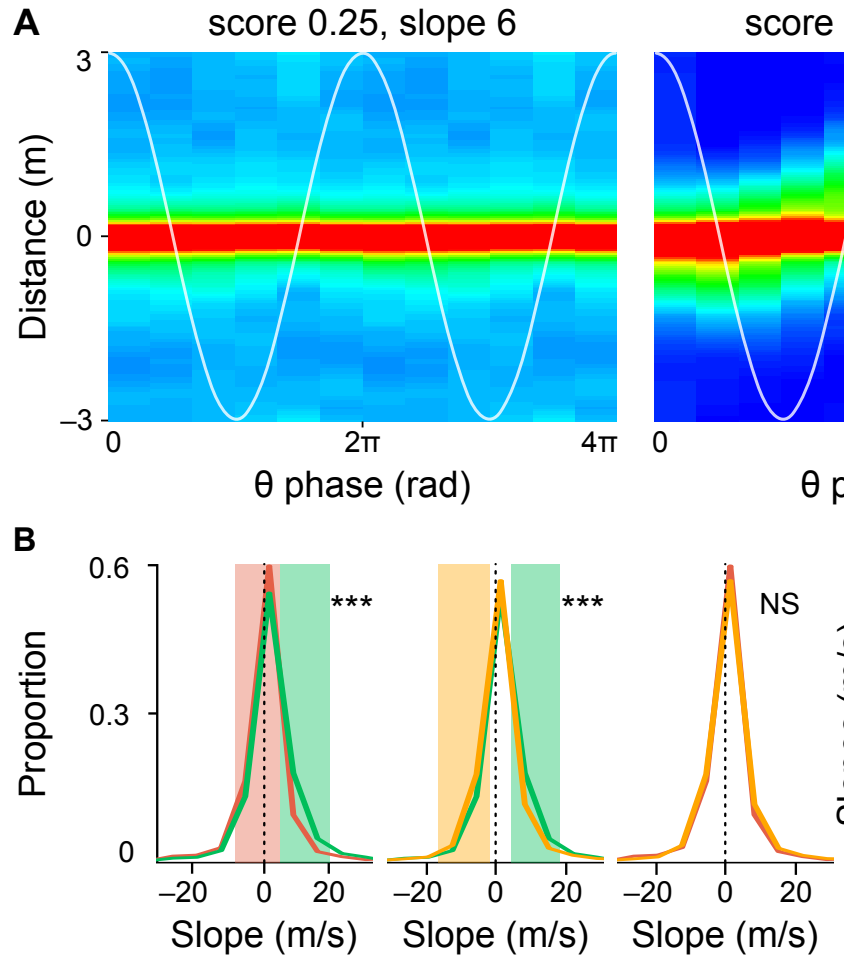

E

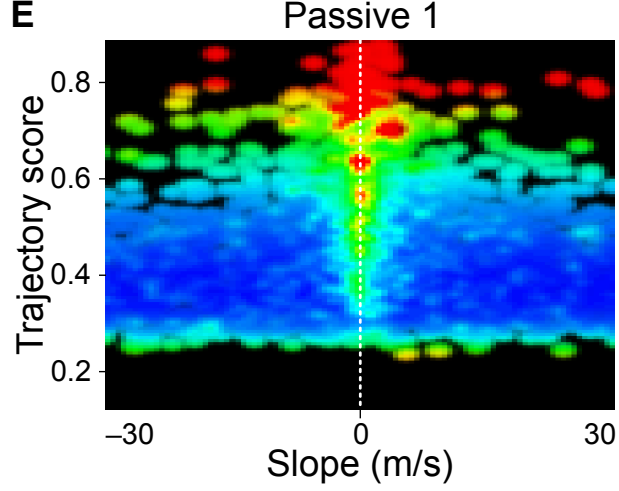

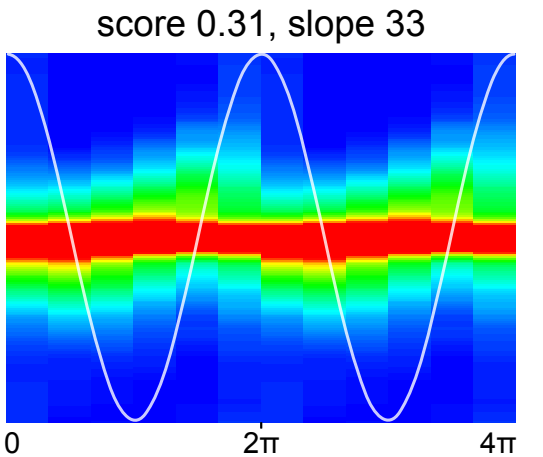

$\theta$ phase (rad)

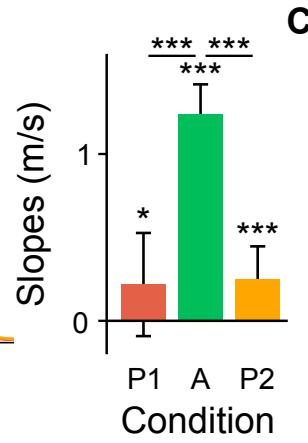

Condition

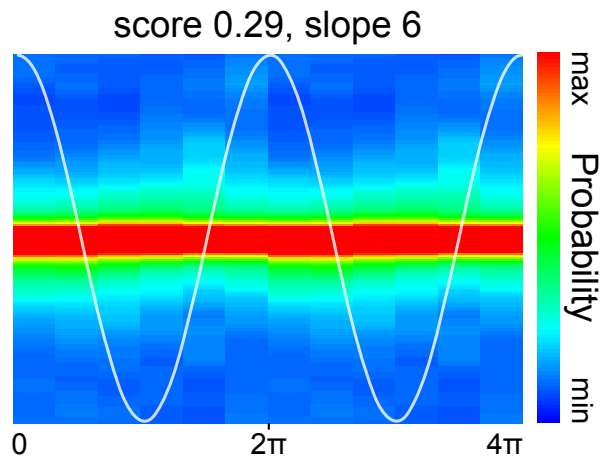

$\theta$ phase (rad)
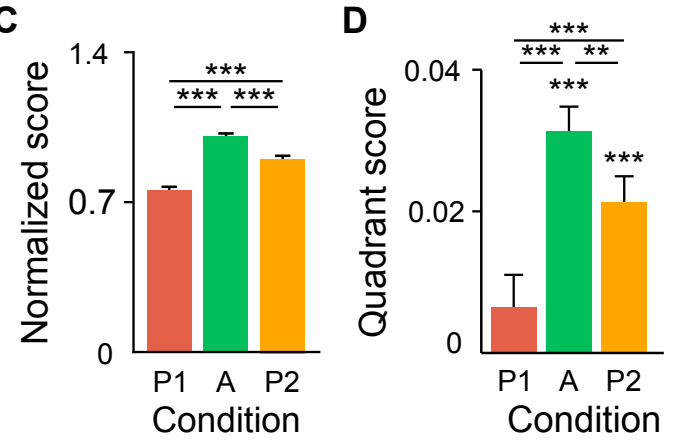

Fig. S10. Trial-to-trial field variability does not account for theta sequence degradation during passive travel. In order to control for firing variability across laps, the Bayesian decoding algorithm was retrained on each individual lap before sequences were assessed. (A) Rat position was estimated across individual theta subcycles and averaged across all rats (same as in fig. 3B). Trajectory scores and slopes (in $\mathrm{cm}$.cycle $\mathrm{C}^{-1}$ ) are indicated above each reconstruction. (B) Distributions of significant theta sequence slopes (KS tests; left: P1-A, ${ }^{* * *} P<10^{-12}$; center: $\mathrm{A}-\mathrm{P} 2,{ }^{* * *} P<10^{-13}$; right: $\left.\mathrm{P} 1-\mathrm{P} 2, P>0.05\right)$. Colored bands indicate significant differences. Right, slopes of significant theta sequences (KW test, ${ }^{* * *} P<10^{-18}$ ). (C) Normalized score of theta sequences (KW test, ${ }^{* * *} P<10^{-27}$ ). (D) Quadrant score computed from individual theta cycles (KW test, ${ }^{* * *} P<10^{-9}$ ). (E) Distribution of theta sequence quality assessed by joint trajectory score and slope (color codes for proportion relative to shuffled control data). 
A

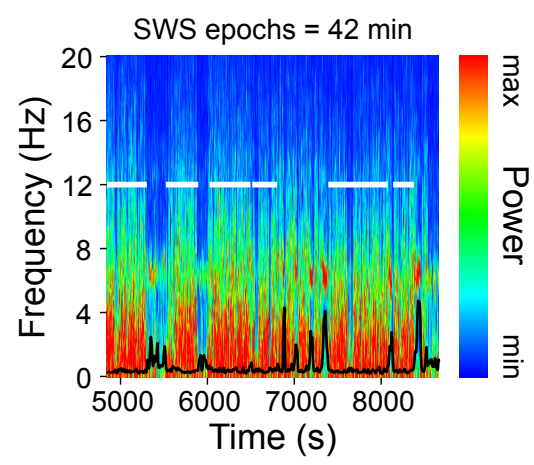

D

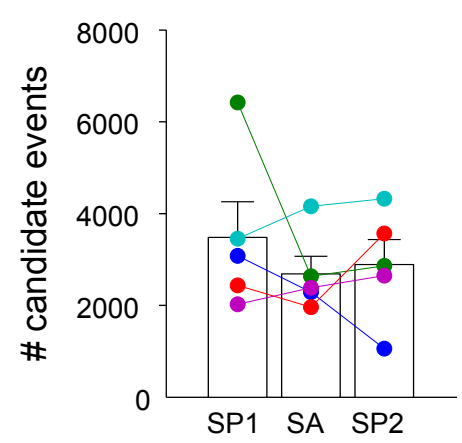

G
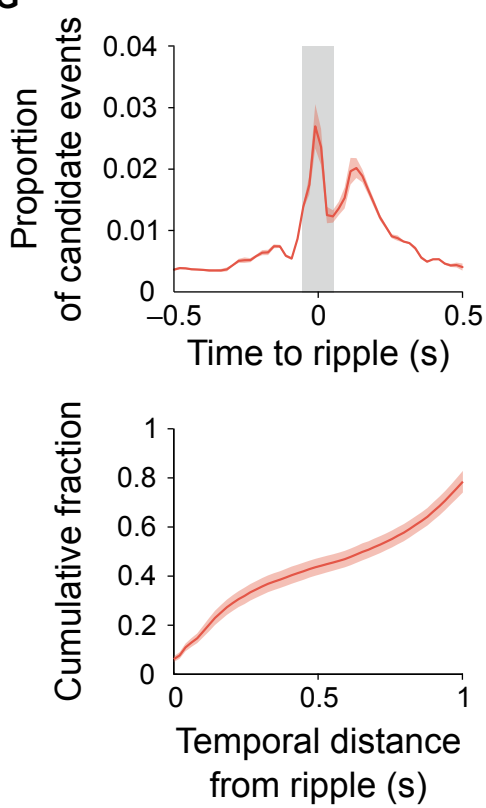

B

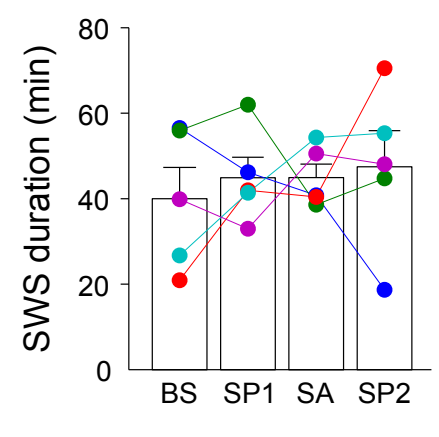

E
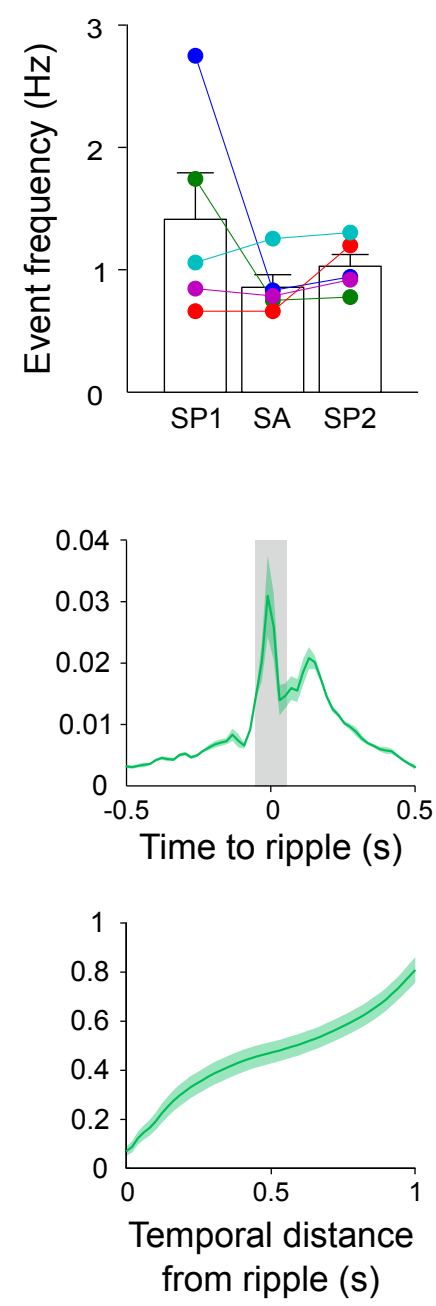

C

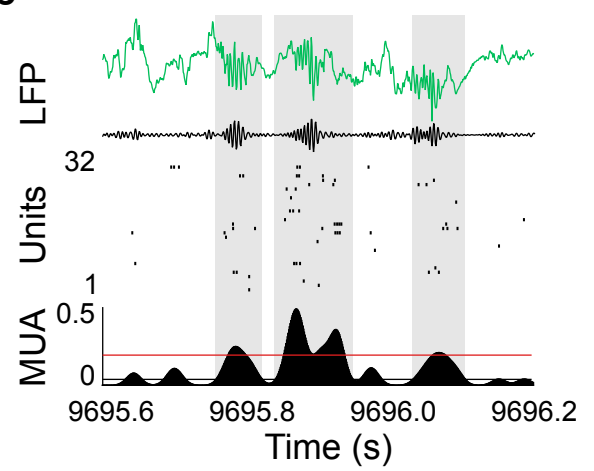

$\mathbf{F}$
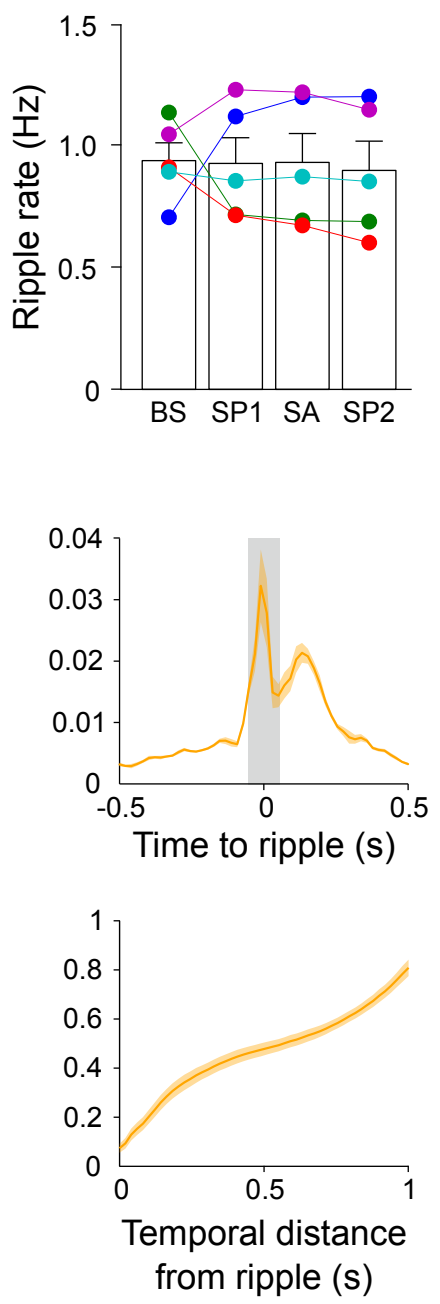

Fig. S11. Candidate replay events during SWS. (A) During sleep recordings, SWS periods were isolated based on the theta/delta ratio (black curve on power spectrogram). K-means clustering identified epochs with the smallest ratio (min duration $120 \mathrm{~s}$, brief interruptions $<1 \mathrm{~s}$ ). (B) SWS duration was not significantly different between sleep conditions (repeated measures ANOVA, $P>0.05$ ). (C) Detection of candidate events for an example SWS period. Top traces, raw (green) and ripple-band filtered (black) LFP. Middle, place cell activity. Cells are ordered according the location of their place field on the track. Bottom, multi-unit activity (MUA) of place cells (time bin, $1 \mathrm{~ms}$; black line, mean of the smoothed histogram, used to define the beginning and end of selected candidates; red line, 3 s.d. above the mean, used as a threshold for the peak). Note that candidate events coincide with ripples.(D-F) The number of candidate events $(\mathbf{D})$, their frequency $(\mathbf{E})$, and ripple rates $(\mathbf{F})$ were not significantly different between sleep conditions (repeated measures ANOVA, $P>0.05$ ). (G) Top, peri-event time histograms of candidate replay events relative to peak ripple power. Grey area indicates the beginning and end of ripples and 0 the time of the peak ripple power (KS test, $P>0.05$ between all condition pairs). Bottom, cumulative distribution of replay events in $\pm 1 \mathrm{~s}$ time windows centered on ripple peak. 
A

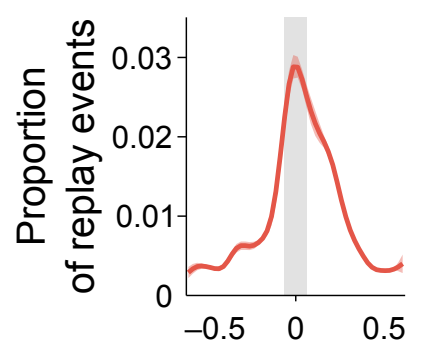

Time to ripples (s)

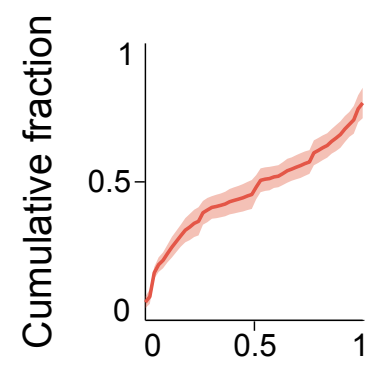

Temporal distance from ripples (s)

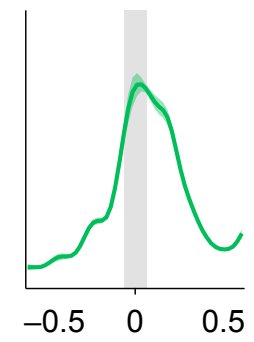

Time to ripples (s)

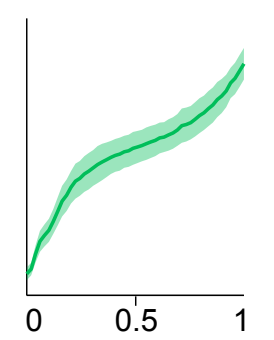

Temporal distance from ripples (s)

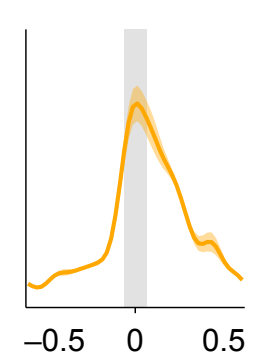

Time to ripples (s)

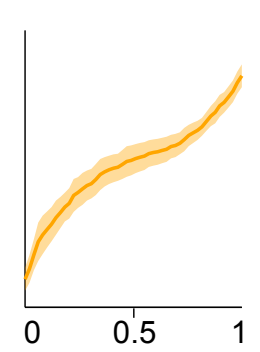

Temporal distance from ripples (s)
B

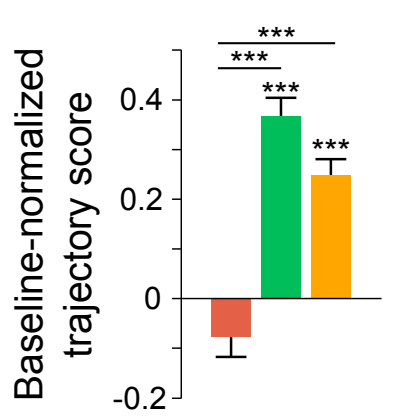

C

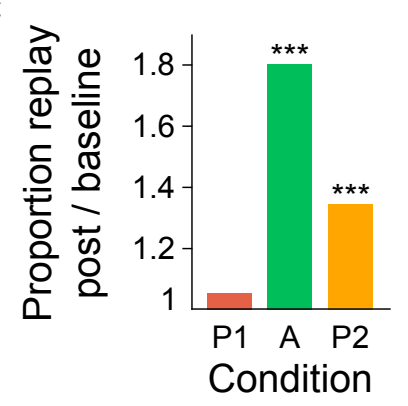

D

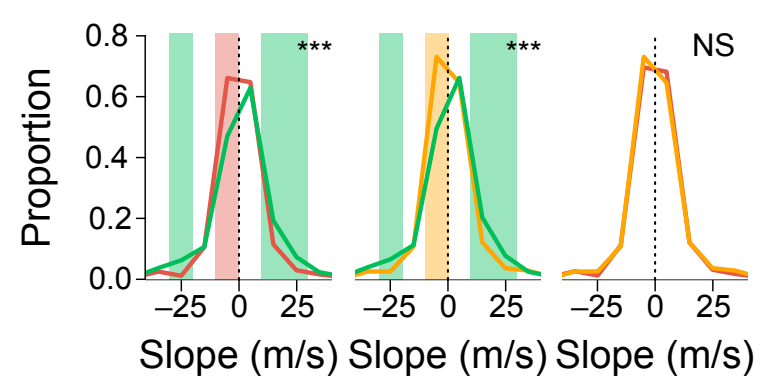

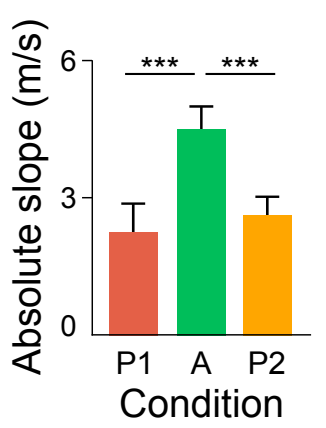

E

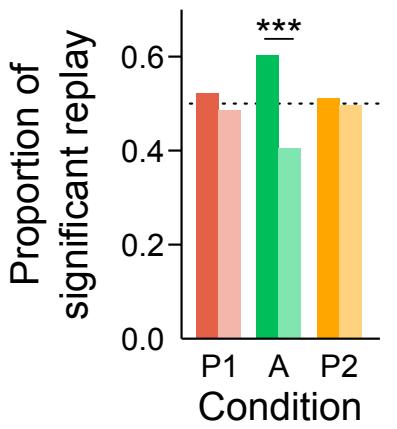

$\mathbf{F}$
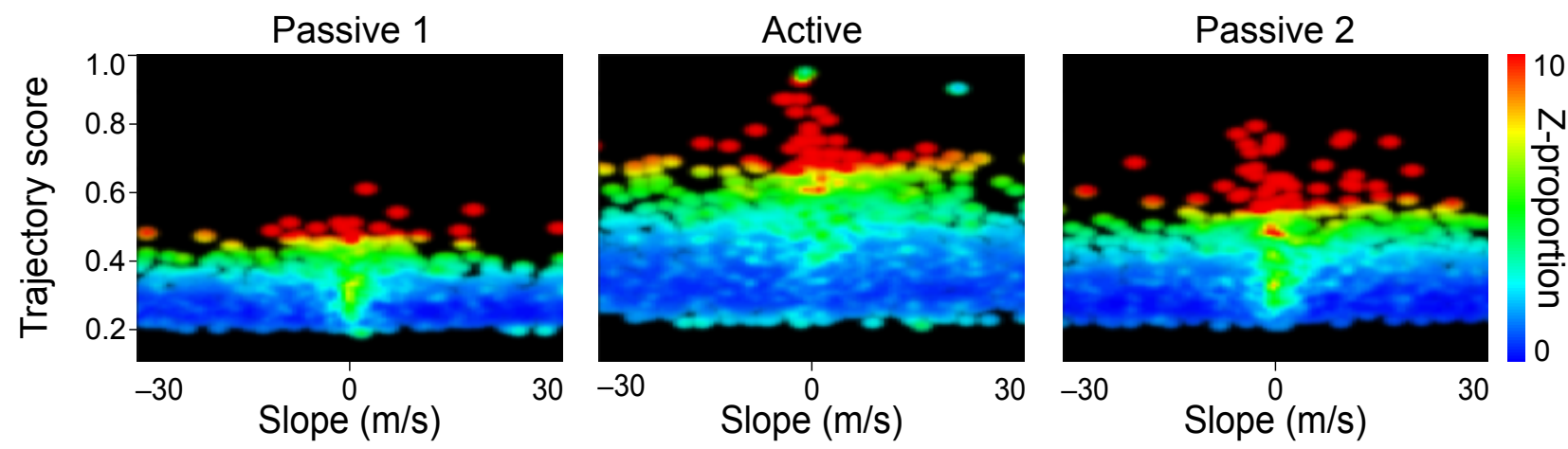

Fig. S12. Restricting analyses to ripple epochs does not alter the results. (A) Top, peri-event time histograms of candidate replay events relative to peak ripple power. Grey area indicates the duration of ripples and 0 the time of peak ripple power (KS test, $P>0.05$ between all condition pairs). Bottom, cumulative distribution of replay events in $\pm 1 \mathrm{~s}$ time windows centered on ripple peak. (B-F) The analyses in fig. 4 were replicated on candidate events restricted to ripple epochs. (B) Scores of replay events relative to baseline sleep (KW test, ${ }^{* * *} P<10^{-15}$ ). (C) Proportion of significant replay events relative to baseline sleep. Sleep replay was boosted following active travel, compared to passive travel. (D) Distributions of replay slopes (KS test; left: SP1-SA, ${ }^{* * *} P<10^{-5}$; center: SA-SP2, ${ }^{* * *} P<10^{-5}$; right: SP1-SP2, $\left.P>0.05\right)$. Colored bands indicate significant differences (binomial proportion test). Right, absolute slopes of replay events (KW test, ${ }^{* * *} P<10^{-27}$ ). (E) Proportion of forward (darker colors) vs reverse (lighter colors) replay events. Only in sleep following active travel was replay biased to reflect actual wake experience. (F) Distribution of replay quality assessed by joint trajectory score and slope (color codes for proportion relative to shuffled control data). 
A
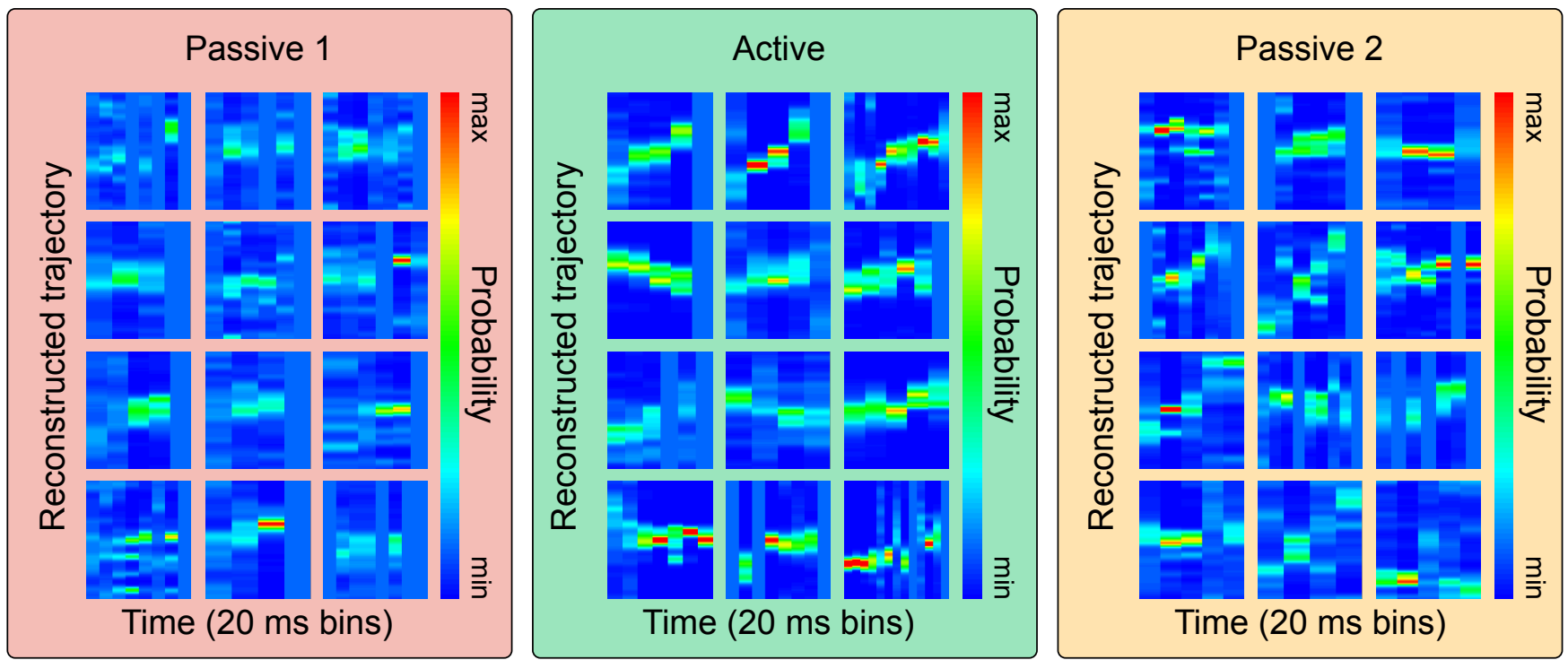

B
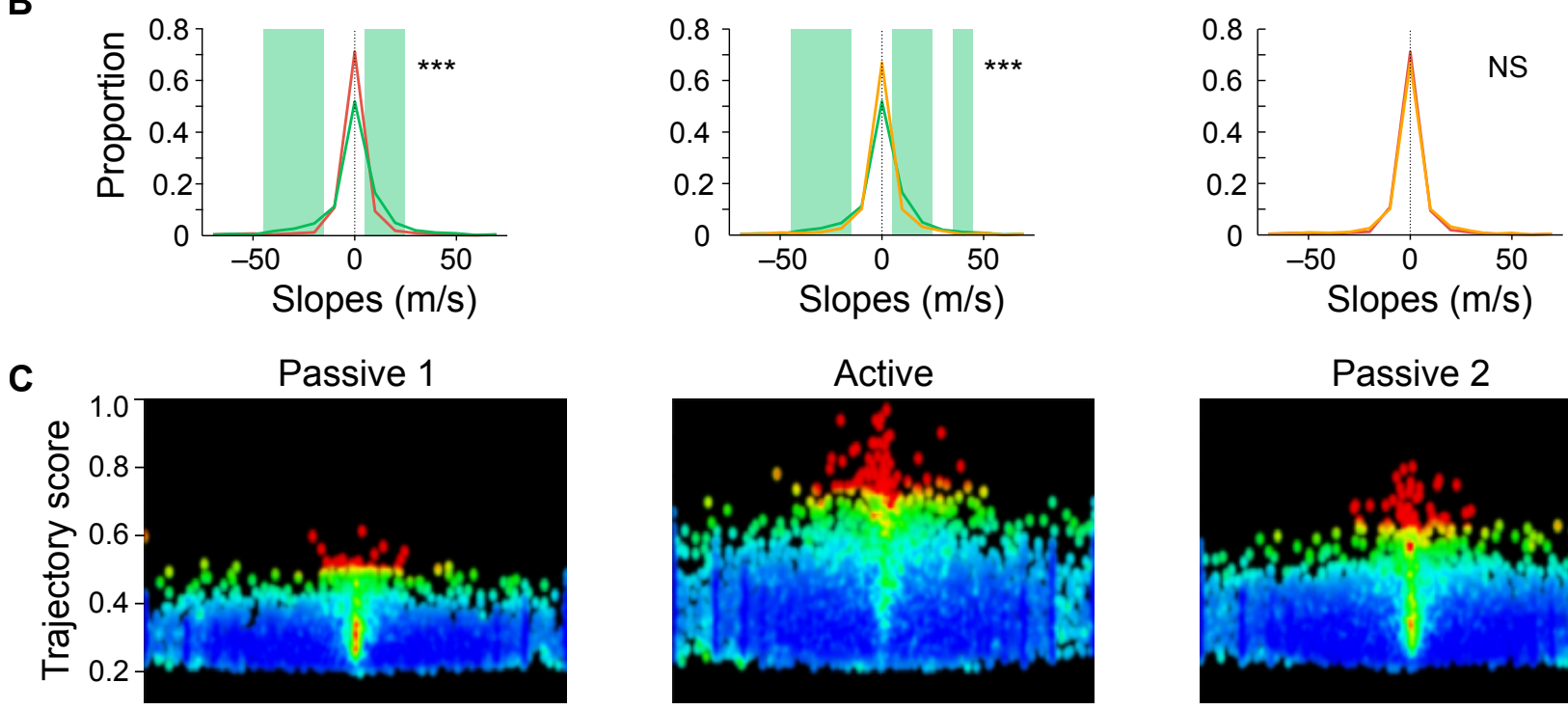

Active
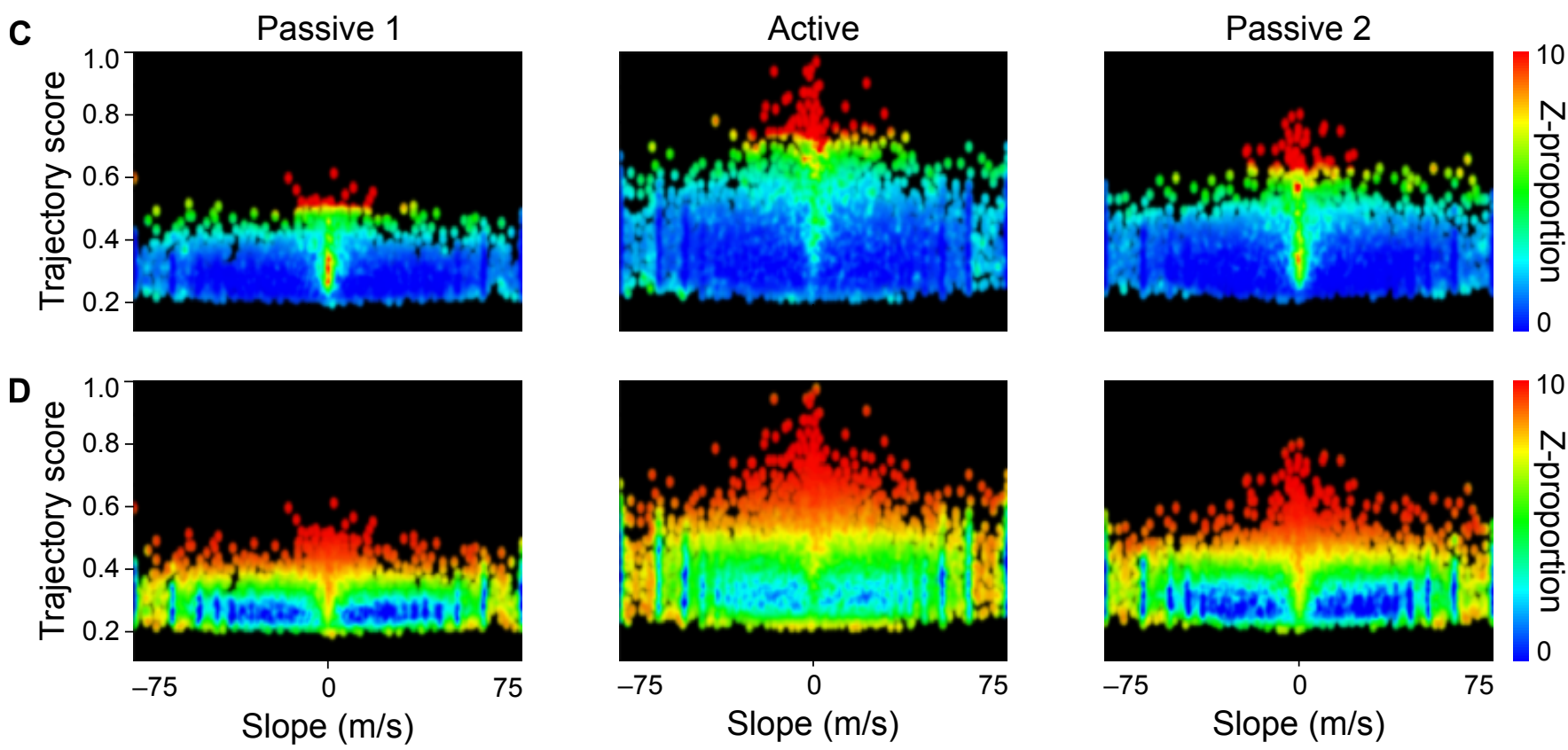

Fig. S13. Replay is degraded following passive travel. (A) Example significant replay events in post-sleep sessions. (B) Distributions of replay slopes (KS tests; left: SP1-SA, ${ }^{* * *} P<10^{-8}$; center: SA-SP2, ${ }^{* * *} P<10^{-6}$; right: SP1-SP2, $P>0.05$ ). Colored bands indicate significant differences. (C-D) Distribution of replay quality assessed by joint trajectory score and slope. (C) Color codes for proportion relative to shuffled control data (same as fig. 4D). (D) Color codes for ratio of observed replay events relative to shuffled control data. 